DRAFT VERSION JuLY 11, 2018

Preprint typeset using $\mathrm{LATEX}_{\mathrm{E}}$ style emulateapj v. 8/13/10

\title{
Spatially-resolved Dense Molecular Gas Excitation in the Nearby LIRG VV 114
}

Toshiki Saito ${ }^{1,2,3}$, Daisuke Iono ${ }^{2,4}$, Daniel Espada ${ }^{2,4}$, Kouichiro NAKAnishi $^{2,4}$, Junko Ueda ${ }^{2,5}$, Hajime Sugai $^{6}$, Min $^{2}$ S. Yun ${ }^{7}$, Shuro Takano ${ }^{8}$, Masatoshi Imanishi ${ }^{9}$, Tomonari Michiyama ${ }^{2,4}$, Satoshi Ohashi ${ }^{10}$, Minju Lee ${ }^{1,2}$, Yoshiaki $^{2}$ Hagiwara $^{11}$, Kentaro Motohara ${ }^{12}$, Takuji Yamashita ${ }^{13}$, Misaki Ando ${ }^{2,4}$ and Ryohei Kawabe ${ }^{1,2,4}$

Draft version July 11, 2018

\begin{abstract}
We present high-resolution observations $\left(0^{\prime \prime} \cdot 2-1^{\prime \prime} .5\right)$ of multiple dense gas tracers, $\mathrm{HCN}$ and $\mathrm{HCO}^{+}$ $(J=1-0,3-2$, and $4-3)$, HNC $(J=1-0)$, and CS $(J=7-6)$ lines, toward the nearby luminous infrared galaxy VV 114 with the Atacama Large Millimeter/submillimeter Array. All lines are robustly detected at the central gaseous filamentary structure including the eastern nucleus and the Overlap region, the collision interface of the progenitors. We found that there is no correlation between star formation efficiency and dense gas fraction, indicating that the amount of dense gas does not simply control star formation in VV 114. We predict the presence of more turbulent and diffuse molecular gas clouds around the Overlap region compared to those at the nuclear region assuming a turbulence-regulated star formation model. The intracloud turbulence at the Overlap region might be excited by galaxy-merger-induced shocks, which also explains the enhancement of gas-phase $\mathrm{CH}_{3} \mathrm{OH}$ abundance previously found there. We also present spatially resolved spectral line energy distributions of $\mathrm{HCN}_{\text {and }} \mathrm{HCO}^{+}$for the first time, and derive excitation parameters by assuming optically-thin and local thermodynamic equilibrium (LTE) conditions. The LTE model revealed that warmer, $\mathrm{HCO}^{+}$ poorer molecular gas medium is dominated around the eastern nucleus, harboring an AGN. The HCN abundance is remarkably flat $\left(\sim 3.5 \times 10^{-9}\right)$ independently of the various environments within the filament of VV 114 (i.e., AGN, star formation, and shock).
\end{abstract}

Subject headings: galaxies: individual (VV 114) — galaxies: interactions — radio lines: galaxies

\section{INTRODUCTION}

$\mathrm{HCN}$ and $\mathrm{HCO}^{+}$are known to be excellent unbiased tracers of extragalactic dense molecular interstellar medium (ISM; e.g., Papadopoulos 2007; Shirley 2015). Rotational transitions of HCN have high optically-thin critical densities $\left(n_{\text {crit }}\right)$ of $\sim 10^{5.5} \mathrm{~cm}^{-3}, 10^{7.0} \mathrm{~cm}^{-3}$, and $10^{7.4} \mathrm{~cm}^{-3}$ at the $J=1-0,3-2$, and $4-3$ transitions, respectively, when assuming collisional excitation with $\mathrm{H}_{2}$ and a kinetic temperature of $20 \mathrm{~K}$. Rotational transitions of $\mathrm{HCO}^{+}$, a molecular ion, also have high optically-

saito@mpia-hd.mpg.de

${ }^{1}$ Department of Astronomy, The University of Tokyo, 7-3-1 Hongo, Bunkyo-ku, Tokyo 113-0033, Japan

2 National Astronomical Observatory of Japan, 2-21-1 Osawa, Mitaka, Tokyo, 181-0015, Japan

${ }^{3}$ Max-Planck Institute for Astronomy, Königstuhl 17, D69117, Heidelberg, Germany

${ }^{4}$ The Graduate University for Advanced Studies (SOKENDAI), 2-21-1 Osawa, Mitaka, Tokyo 181-0015, Japan

${ }^{5}$ Harvard-Smithsonian Center for Astrophysics, 60 Garden Street, Cambridge, MA 02138, USA

${ }^{6}$ Kavli Institute for the Physics and Mathematics of the Universe (WPI), The University of Tokyo, 5-1-5 Kashiwanoha, Kashiwa, 277-8583, Chiba, Japan

${ }^{7}$ Department of Astronomy, University of Massachusetts, Amherst, MA 01003, USA

${ }^{8}$ Physics Department, College of Engineering, Nihon University, 1 Nakagawara, Tokusada, Tamura, Koriyama, Fukushima, 963-8642, Japan

9 Subaru Telescope, 650 North Aohoku Place, Hilo, Hawaii, 96720, USA

10 RIKEN, 2-1, Hirosawa, Wako-shi, Saitama 351-0198, Japan

11 Natural Science Laboratory, Toyo University, 5-28-20, Hakusan, Bunkyo-ku, Tokyo 112-8606, Japan

${ }^{12}$ Institute of Astronomy, The University of Tokyo, 2-21-1 Osawa, Mitaka, Tokyo 181-0015, Japan

${ }^{13}$ Research Center for Space and Cosmic Evolution, Ehime University, 2-5 Bunkyo-ku, Matsuyama, Ehime 790-8577, japan thin $n_{\text {crit }}$ of $\sim 10^{4.7} \mathrm{~cm}^{-3}, 10^{6.1} \mathrm{~cm}^{-3}$, and $10^{6.5} \mathrm{~cm}^{-3}$ at $J=1-0,3-2$, and $4-3$, respectively. A tight linear correlation in $\log$ scale between far-infrared luminosity, $L_{\text {FIR }}$ (or star formation rate, SFR), and luminosity of $\mathrm{HCN}$ or $\mathrm{HCO}^{+}$lines, $L_{\text {dense }}^{\prime}$ (or dense gas mass, $\left.M_{\text {dense }}\right)$ was found in nearby spiral galaxies and (ultra-)luminous infrared galaxies (U/LIRGs) (e.g., Gao \& Solomon 2004a,b; Graciá-Carpio et al. 2008; GarcíaBurillo et al. 2012; Zhang et al. 2014; Privon et al. 2015; Usero et al. 2015; Stephens et al. 2016), suggesting that galaxies have a nearly constant SFR per $M_{\text {dense }}$ (i.e., a constant dense gas star formation efficiency; $\mathrm{SFE}_{\text {dense }}$ ). Even if including local star-forming clouds, the relation is still almost linear (e.g., Shimajiri et al. 2017). Numerical simulations succeeded in reproducing such a linear correlation between $\log L_{\text {FIR }}$ and $\log L_{\text {dense }}^{\prime}$ (e.g., Narayanan et al. 2008). However, Michiyama et al. (2016) found a super-linear relation using $\mathrm{CO}(J=3-2)$ line in gas-rich merging galaxies (hereafter $\mathrm{CO}(3-2)$ line).

For multiple CO transitions in U/LIRGs, Greve et al. (2014) found a decreasing trend of the $L_{\mathrm{FIR}}-L_{\mathrm{CO}}^{\prime}$ index as $J$ increases at least up to $J=13-12$, which can be explained by a substantial contribution of additional warm and dense gas components to the higher- $J$ line luminosities possibly (mechanically) heated by supernovae, stellar winds and/or active galactic nucleus (AGN) outflow (see also Kamenetzky et al. 2016). On the other hand, the relatively weaker intensities of $\mathrm{HCN}$ and $\mathrm{HCO}^{+}$transitions make it harder in general to study such $J$ dependence for these dense gas tracers for individual galaxies (and from region to region).

Contrary to the analysis of excitation conditions of some molecular species (mostly CO), molecular line in- 

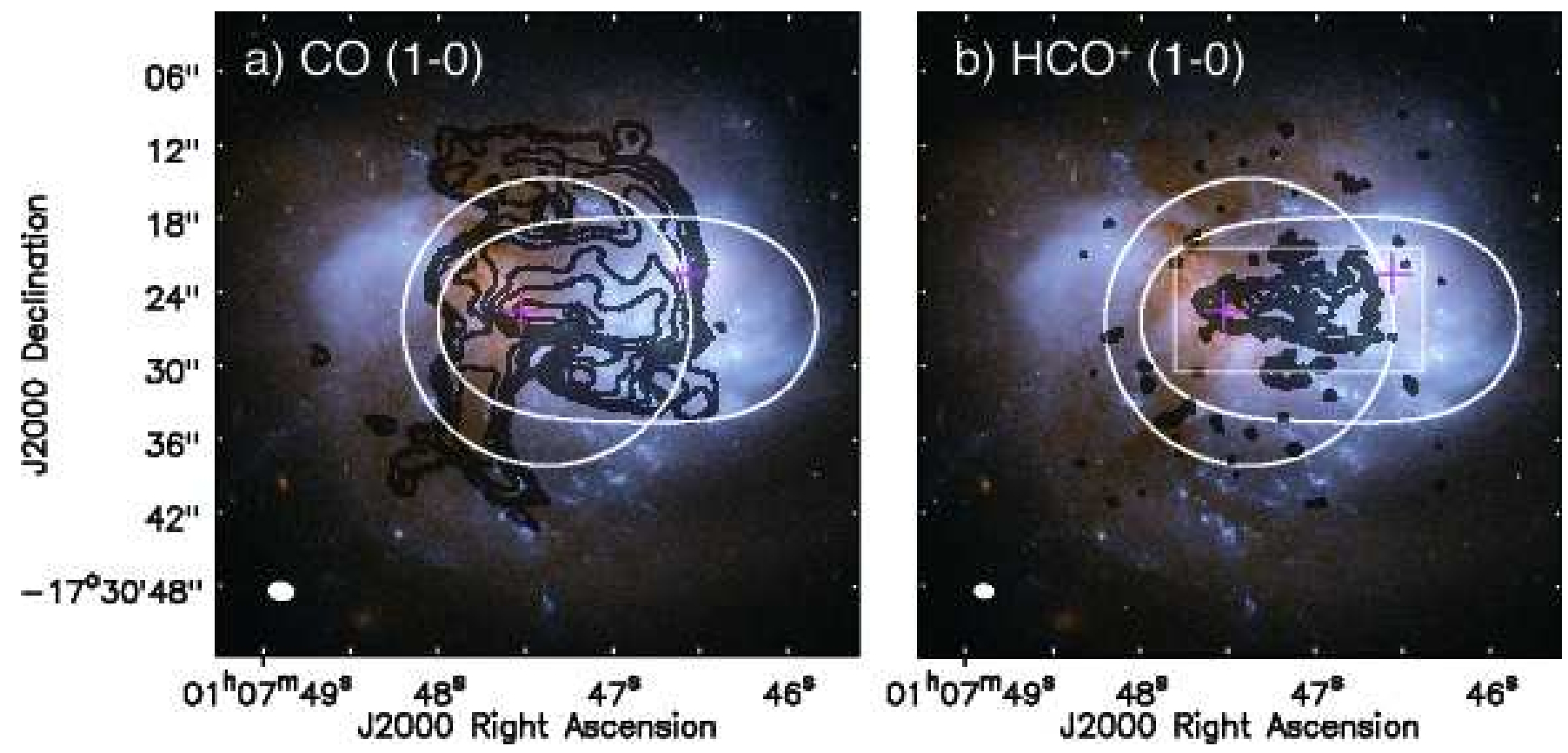

Figure 1. (a) ALMA CO (1-0) contours (Saito et al. 2015) overlaid on the HST/ACS image of VV 114 [Credit: NASA, ESA, the Hubble Heritage (STScI/AURA)-ESA/Hubble Collaboration, and A. Evans (University of Virginia, Charlottesville/NRAO/Stony Brook University]. The contours are $0.2,0.4,0.8,1.6,3.2,6.4,12.8,25.6$, and $33.0 \mathrm{Jy}$ beam $^{-1} \mathrm{~km} \mathrm{~s}^{-1}$. The white circle and ellipse show the field of view of Band 6 and Band 7, respectively. The magenta crosses show the positions of the nuclei defined by the peak positions of the 1." 0 resolution Ks-band image (Tateuchi et al. 2015). (b) Same as (a), but for $\mathrm{HCO}^{+}(1-0)$. The contours are $0.67 \times(0.04,0.08,0.16,0.32$, 0.64 , and 0.96$) \mathrm{Jy} \mathrm{beam}^{-1} \mathrm{~km} \mathrm{~s}^{-1}$.

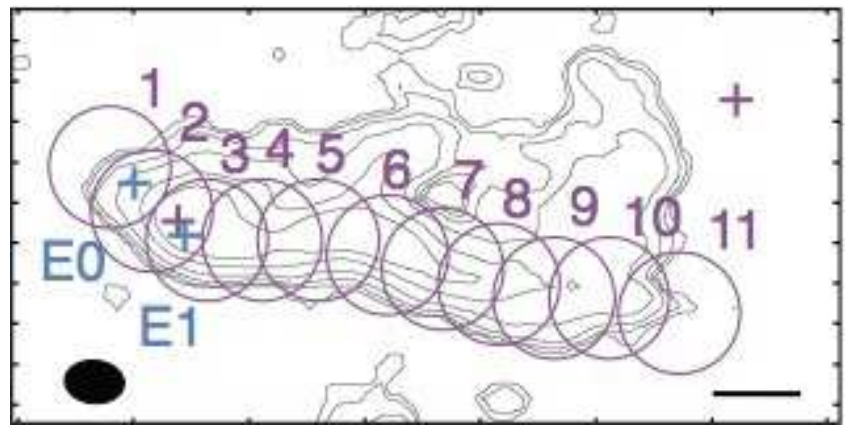

Figure 2. The central region of VV 114 as seen in $\mathrm{HCO}^{+}(1-0)$ emission with the aperture and position names used for the flux measurements in this paper; E0 and E1 are the AGN and starburst (cyan crosses) as defined by Iono et al. (2013) and Region 1-11 are apertures along the central dust filament defined by Saito et al. (2017b). The magenta crosses show the positions of the nuclei defined by the peak positions of the $1{ }^{\prime \prime} 0$ resolution Ks-band image (Tateuchi et al. 2015). The scale bar corresponds to $2^{\prime \prime} 0$ (= $\left.840 \mathrm{pc}\right)$.

tensity ratios between different species have been broadly studied for nearby starburst galaxies and U/LIRGs (Aalto 2013, and references therein). Some ratios of bright molecular lines have been proposed to be used to diagnose physical and chemical processes involved in starburst and $\mathrm{AGN}$ hidden inside the dusty nuclear regions (e.g., ${ }^{12} \mathrm{CO} /{ }^{13} \mathrm{CO}, \mathrm{HCN} / \mathrm{CO}, \mathrm{HCN} / \mathrm{HCO}^{+}$, $\mathrm{HCN} / \mathrm{HNC}, \mathrm{CN} / \mathrm{HCN}$; Aalto et al. 1991; Kohno et al. 2001; Aalto et al. 2002; Gao \& Solomon 2004a,b; Meier \& Turner 2005; Costagliola et al. 2013). In particular, the $\mathrm{HCN} / \mathrm{HCO}^{+}$line intensity ratios for a given $J\left(J_{\text {upp }}\right.$ $<5)$ are among the most established diagnostic tracers of AGN in $\mathrm{mm}$ and sub-mm wavelengths, and observational case studies of the ratios have been published for some bright galaxies (Kohno et al. 2001; Kohno 2005; GraciáCarpio et al. 2006; Imanishi et al. 2007, 2016a,b,c, 2018; Knudsen et al. 2007; Papadopoulos 2007; Krips et al. 2008; Costagliola et al. 2011; Hsieh et al. 2012; Imanishi \& Nakanishi 2013a,b, 2014; Iono et al. 2013; Izumi et al. 2013, 2015, 2016; García-Burillo et al. 2014; Aalto et al. 2015a; Aladro et al. 2015; Martín et al. 2015; Saito et al. 2015; Schirm et al. 2016; Ueda et al. 2016; Espada et al. 2017; Salak et al. 2018). A few observational and theoretical studies address the excitation state of both molecules (e.g., Krips et al. 2008; Meijerink et al. 2011; Izumi et al. 2013; Papadopoulos et al. 2014; Spilker et al. 2014; Kazandjian et al. 2015; Tunnard et al. 2015).

Understanding the excitation conditions of the dense molecular gas is a natural step to investigate the relation between dense gas, star formation, and AGN. U/LIRGs are ideal sources to study the excitation of the HCN and $\mathrm{HCO}^{+}$lines because the high $L_{\mathrm{FIR}}$ suggests that dense material is abundant near the nuclear region of these galaxies, and even the high- $J$ transitions of dense gas tracers can be detected with relatively short integration times. Accordingly, we have used ALMA in the past years to study the dense gas in the merging galaxy $\mathrm{VV}$ 114 (Iono et al. 2013; Saito et al. 2015, 2017a, but see also previous studies with other interferometers, Yun et al. 1994; Iono et al. 2004a; Wilson et al. 2008; Sliwa et al. 2013).

The IR-bright mid-stage merger VV $114\left(D_{\mathrm{L}}=87\right.$ Mpc, $L_{\mathrm{IR}}=10^{11.69} L_{\odot}, 1^{\prime \prime}=420$ pc; Armus et al. 2009$)$ is an intriguing system especially for the $\mathrm{HCN}$ and $\mathrm{HCO}^{+}$ lines. Figure 1a shows the ALMA CO $(1-0)$ image $\left(\sim 1^{\prime \prime} .5\right.$ resolution) of VV 114 overlaid on the stellar light taken by $H S T /$ ACS F814W (Saito et al. 2015). The CO emission mainly comes from the central part of VV 114, and 
the peaks do not coincide with the K-band nuclei (shown as magenta crosses in Figure 1). The dense gas in VV 114 was first imaged in the $\mathrm{HCN}(1-0)$ and $\mathrm{HCO}^{+}(1-0)$ lines with Nobeyama Millimeter Array (5-7"'resolution; Imanishi et al. 2007). The $\mathrm{HCN}(1-0) / \mathrm{HCO}^{+}(1-0)$ flux density ratio is higher than 1.6 at the eastern galaxy. Iono et al. (2013) identified a compact (unresolved by $\sim 0.5$ resolution $)$, broad $\left(\sim 290 \mathrm{~km} \mathrm{~s}^{-1}\right)$ component in $\mathrm{HCN}(4-3)$ and $\mathrm{HCO}^{+}(4-3)$ lines at the eastern nucleus indicating the presence of a massive object there $(\lesssim 4 \times$ $\left.10^{8} M_{\odot}\right)$. The unresolved massive object (E0) and the brightest dense gas clump (E1) identified by Iono et al. (2013) coincide with the two strongest K-band peaks at the eastern nucleus (Scoville et al. 2000; Tateuchi et al. 2015) This unresolved component was also detected by using Submillimeter Array observations of the $\mathrm{HCO}^{+}(4-$ 3) line (Wilson et al. 2008). The $\mathrm{HCN}(4-3) / \mathrm{HCO}^{+}(4-3)$ line ratio at the eastern nucleus was higher than unity, whereas other star-forming dense gas clumps along the central kpc-scale filament of VV 114 showed lower values $(<1)$. Combining this information with the observed characteristics in other wavelengths (e.g., Paschen $\alpha$ and hard X-ray; Grimes et al. 2006; Tateuchi et al. 2015), Iono et al. (2013) suggested that the eastern nucleus of VV 114 may harbor a dusty AGN (see Figure 2), which coincides with a hard X-ray point source (Grimes et al. 2006). Contrary to the eastern nuclei, through our followup $\mathrm{CH}_{3} \mathrm{OH}$ observations (Saito et al. 2017a) we found that the Overlap region, which is located between the progenitor's disks of the VV 114 system (i.e., the western side of the kpc-scale filament), is affected by mergerinduced shocks. In summary, VV 114 has three distinctive regions that are expected to show different dense gas excitation and/or chemistry, i.e., the X-ray-bright AGN, starburst regions, and the shocked Overlap, and thus it is a good target to study the properties of dense gas ISM under different environments in a LIRG. VV 114 is known as the closest analogue of high-z Lyman break galaxies (LBGs) due to its FUV characteristics (Grimes et al. 2006). Since LBGs are thought to constitute a substantial fraction of star-forming galaxies during $2 \leq \mathrm{z} \leq$ 6 (Peacock et al. 2000), it is also important to inspect the ISM properties in objects such as VV 114 in order to understand the onset of star formation at high redshift.

This Paper is organized as follows. Section 2 describes a brief summary of our ALMA observations toward VV 114 and the procedure of data reduction. All $\mathrm{HCN}$ and $\mathrm{HCO}^{+}$lines and continuum images are presented in Section 3. Then, we present beam- and $u v$ matched line ratio images in Section 4. In Section 5, we discuss the dense gas-FIR luminosity relations and their $J$ dependences (Section 5.1), star-forming activities using SFEs and dense gas fractions (Section 5.2) and its modeling (Section 5.3), line ratios against SFR (Section 5.4), and the physical properties of dense gas using a radiative transfer model (Section 5.5). Finally, we summarize our main findings in Section 6. We have adopted $\mathrm{H}_{0}=70$ $\mathrm{km} \mathrm{s}^{-1} \mathrm{Mpc}^{-1}, \Omega_{m}=0.3$, and $\Omega_{\Lambda}=0.7$ throughout this Paper.

\section{OBSERVATIONS AND DATA REDUCTION}

2.1. Cycle 2 ALMA: $J=1-0$
The Band 3 line survey toward VV 114 was carried out during the ALMA cycle 2 period (ID: 2013.1.01057.S, PI: T. Saito). Although the aim of this project was an unbiased line survey $(84-111 \mathrm{GHz}$ and $127-154 \mathrm{GHz})$ to study the chemistry in the filament of VV 114, here we only present two tunings which include the bright dense gas tracers, $\mathrm{HCN}(1-0)\left(\nu_{\mathrm{obs}}=86.88826 \mathrm{GHz}\right)$, $\mathrm{HCO}^{+}(1-0)\left(\nu_{\mathrm{obs}}=87.43399 \mathrm{GHz}\right)$, and the ground transition of an isomer of hydrogen cyanide, HNC (1-0), $\left(\nu_{\mathrm{obs}}=88.88001 \mathrm{GHz}\right)$ lines. The full description of this project will be provided in a forthcoming paper.

A tuning (B3-1) covers the $\mathrm{HCN}(1-0)$ and $\mathrm{HCO}^{+}(1-$ 0 ) lines in the lower sideband, and another tuning (B32) also covers the HNC (1-0) line in the lower sideband. B3-1 (B3-2) was obtained on 2014 June 17, July 2, 2015 June 4, and 5 (2014 July 3 ) with a single-sideband system temperature $\left(T_{\text {sys }}\right)$ of $38-90 \mathrm{~K}(33-78 \mathrm{~K})$. The antenna configuration for B3-1 (B3-2) had thirty to thirty-eight (thirty-one) $12 \mathrm{~m}$ antennas, with a projected baseline length ( $\left.L_{\text {baseline }}\right)$ of 18-780 m (19-650 m), which corresponds to a maximum recoverable scale (MRS; Lundgren et al. 2013) of $\sim 22^{\prime \prime}$. Each tuning had four spectral windows (spws) to cover both sidebands. Each spw had a bandwidth of $1.875 \mathrm{GHz}$ and $1.938 \mathrm{MHz}$ resolution. The total on-source time of B3-1 (B3-2) was 47.4 minutes (23.7 minutes). The field of view (FoV) of Band 3 covers most of the extended CO (1-0) emission (Figure 1a). Neptune or Uranus were used as flux calibrators, while J0137-2430 or J2258-2758 were used as bandpass calibrators for both tunings. J0110-0741 or J0116-2052 were observed as phase calibrators.

$$
\text { 2.2. Cycle 3 ALMA: J = 3-2 }
$$

Band 6 observations toward VV 114 were carried out during the ALMA cycle 3 period (ID: 2015.1.00973.S, PI: T. Saito). The upper sideband was tuned to cover the $\mathrm{HCN}(3-2)$ and $\mathrm{HCO}^{+}(3-2)$ lines. The data was obtained on 2016 May 23 with $T_{\text {sys }}$ of $62-180 \mathrm{~K}$. The assigned configuration had thirty-seven $12 \mathrm{~m}$ antennas with $L_{\text {baseline }}$ of $16.7-641.5 \mathrm{~m}$ (MRS $\left.\sim 9^{\prime \prime}\right)$. Each tuning had four spws to cover both sidebands. Two of the spws including the target lines had a bandwidth of $1.875 \mathrm{GHz}$ with $7.812 \mathrm{MHz}$ resolution, whereas the other two have a bandwidth of $2.000 \mathrm{GHz}$ with $15.625 \mathrm{MHz}$ resolution. The total on-source time was 30.4 minutes. The FoV of Band 6 covers all structures found in the $\mathrm{HCO}^{+}(1-0)$ (Figure 1b). Pallas, J0006-0623, J0118-2141 were used as the flux, bandpass, and phase calibrators, respectively. However, we estimate the absolute flux scaling factor using the bandpass calibrator, because the visibility model of asteroids in CASA is not reliable currently (see Section 2.4).

$$
\text { 2.3. Cycle 2 ALMA: J = 4-3 }
$$

VV 114 was observed with the long baseline mode of cycle 2 ALMA at Band 7 (ID: 2013.1.00740.S, PI: T. Saito). The field and frequency setups of the long baseline observations are the same as for our previous $\mathrm{HCN}(4-3)$ and $\mathrm{HCO}^{+}$(4-3) observations with ALMA (Iono et al. 2013; Saito et al. 2015) except for the channel width (976.562 kHz for this Cycle 2 data). The lower sideband was tuned to cover the CS (7-6) line. The data were obtained on 2015 June 28 (B7-1) and July 18 
(B7-2) with $T_{\text {sys }}$ of 75-205 and 90-310 K, respectively. The assigned array configuration of B7-1 and B7-2 had fourty-one $12 \mathrm{~m}$ antennas with $L_{\text {baseline }}$ of $43.3 \mathrm{~m}-1.6 \mathrm{~km}$ and thirty-eight $12 \mathrm{~m}$ antennas with $L_{\text {baseline }}$ of $15.1 \mathrm{~m}-$ $1.6 \mathrm{~km}$, respectively. The combined data has MRS of $7^{\prime \prime}$. Each tuning has four spws to cover both sidebands. All spws have a bandwidth of $1.875 \mathrm{GHz}$ with $976.562 \mathrm{kHz}$ resolution. The total on-source time of each data is $\sim 41.2$ minutes. J2258-279, J2348-1631, and J0132-1654 were used as the flux, bandpass, and phase calibrators for both data, respectively.

\subsection{Data Reduction}

Processing the new Band 3, Band 6, and Band 7 data, including calibration and imaging, was done using CASA version 4.2.2, 4.5.3, and 4.2.2, respectively (McMullin et al. 2007). Images were reconstructed with the natural (robust $=2.0$ ) or briggs (robust $=0.5$ ) weighting. We made the data cubes with a velocity resolution of 20 , 30 , or $50 \mathrm{~km} \mathrm{~s}^{-1}$ depending on achieved noise rms and signal-to-noise ratio of the target lines. Continuum emission was subtracted in the $u v$-plane by fitting the line free channels in both USB and LSB with a first order polynomial function. The line-free channels were used to make a continuum image using the multi-frequency synthesis method. Imaging properties, including flux density and recovered flux (i.e., ALMA interferometric flux relative to flux measured by a single-dish telescope), are listed in Table 1 . We roughly recovered half of the total fluxes measured by single dish telescopes. All images shown in this Paper, except for line ratios, are not corrected for primary beam attenuation.

We use $\mathrm{HCN}(4-3)$ and $\mathrm{HCO}^{+}(4-3)$ data taken in ALMA cycle 0 (ID: 2011.0.00467.S, PI: D. Iono; Iono et al. 2013; Saito et al. 2015), and combine them with the cycle 2 data (Section 2.3). In those previous papers, we carried out the absolute flux calibrations with the Butler-Horizons-2010 (BH10) models. However, the flux models were updated, the so-called Butler-JPL-Horizons 2012 (BJH12), so we corrected the previous HCN (4-3) and $\mathrm{HCO}^{+}(4-3)$ fluxes using the new models following the description in the CASA guides ${ }^{14}$. The flux calibrator used for the cycle 0 data was Uranus, and the difference of the "zero-spacing" flux density of Uranus between the models (i.e., BJH2012/BH2010 - 1) at $349 \mathrm{GHz}$ is +0.03 . Therefore, we multiplied the flux densities of the cycle 0 data by 1.03 before combination. Furthermore, we used the CASA task statwt ${ }^{15}$ to recalculate the visibility weights of the cycle 0 data.

The systematic error on the absolute flux scaling factor using a solar system object is $5 \%$ for the Band 3 data (Lundgren et al. 2013). As described in Section 2.2, we used the bandpass calibrator as the flux calibrator for the Band 6 data in order to avoid using the unreliable flux model of Pallas. Using the ALMA Calibrator Source Catalogue $^{16}$, the flux uncertainty of J0006-0623 at 260 $\mathrm{GHz}$ on 2016 May 23 is estimated to be $7.9 \%$. This was estimated by fitting the monitored measurement at 91.5 ,

\footnotetext{
${ }^{14}$ https://casaguides.nrao.edu/index.php/Solar_System_Models_ in_CASA_4.0

15 https://casaguides.nrao.edu/index.php/DataWeightsAndComb ination

16 https://almascience.nao.ac.jp/sc/
}

103.5 and $343.5 \mathrm{GHz}$ on 2016 May 25 using $S_{\nu} \propto \nu^{\alpha}$, where $S_{\nu}$ is the flux density, $\nu$ is the observed frequency, and $\alpha$ is the spectral index. The derived $\alpha$ of J0006-0623 is $-0.48 \pm 0.01$. Therefore, we adopt a flux uncertainty of the Band 6 data as $8 \%$ throughout this Paper. Since the flux uncertainties of J2258-279 at $343.5 \mathrm{GHz}$ (i.e., new Band 7 data) on 2015 June 29 and July 20 are $\sim 7.7 \%$ and $15.4 \%$, respectively, we adopt the flux uncertainty of $15 \%$ for the Band 7 data.

If flux measurements for our bandpass and phase calibrators in less than a week from the observing date are available in the catalogue, we check the validity of the absolute flux calibrations using their fluxes for each spw. For the B3-1 data, the observed fluxes and the monitored values of J0116-2052 and J2258-2758 are consistent $(<9 \%)$. For the B3-2 data, the flux differences of J0116-2052 are less than 6\%. The flux differences of J0118-2141, which was used for the Band 6 observation, are less than $30 \%$. This is a relatively large value probably because uncertain flux interpolation between Band 3 and Band 7. The calibrators and check source observed in the Band 7 data were not monitored within 154 days from our observing dates.

MRS of each data is different due to the differences of the observed frequency and the minimum $L_{\text {baseline }}$. When we discuss line ratios between data which have different MRS, we clipped all visibility data inside $18 k \lambda$ in order to achieve a similar MRS, which allows us to minimize different missing flux effects. Beam convolution to 1 .'5 is also performed after $u v$-clipping.

\section{RESULTS}

The $254 \mathrm{GHz}$ (Band 6) and $350 \mathrm{GHz}$ (Band 7) continuum images are shown in Figures $3 \mathrm{a}$ and $3 \mathrm{~b}$, respectively. The total flux of VV 114 at $260 \mathrm{GHz}$ and $350 \mathrm{GHz}$ are $17.4 \pm 1.4 \mathrm{mJy}$ and $44.7 \pm 6.7 \mathrm{mJy}$, respectively. Their overall spatial distributions along the filament across VV 114 coincide with each other and also with previous $340 \mathrm{GHz}$ (i.e., dust emission mostly) continuum images (Wilson et al. 2008; Saito et al. 2015). They also agree with the low-resolution $110 \mathrm{GHz}$ (i.e., mostly free-free) (Saito et al. 2015) and the $1.4 \mathrm{GHz}$ (i.e., synchrotron) radio continuum image (Yun et al. 1994). The filamentary structure consists of a dozen of clumps. The easternmost point-like source in the filament coincides with the putative AGN (Iono et al. 2013).

The integrated intensity, velocity field, and velocity dispersion images of all transitions are shown in Figures 4,5 , and 6 . All lines are mainly detected at the central filamentary structure which was identified in many molecular lines as well as ionized gas tracers (Tateuchi et al. 2015), indicating a site of intense star formation, as already seen in the radio-to-FIR continuum emissions. The filament consists of three main blobs located at the eastern nucleus, which may harbor a putative AGN (E0, see Figure 2) and starbursting clumps (E1), and between the progenitor's disks ("Overlap" region). For a given $J$, the $\mathrm{HCO}^{+}$emission is more extended and brighter than the HCN emission. HNC (1-0) has two peaks, one at E0 and the another at E1 (see also Section 3.2). Although the clumps in the eastern galaxy reported by Iono et al. (2013) are clearly resolved in the Band 6 and Band 7 data, they are only marginally resolved in the Band 3 data due to the coarser beam size. The velocity field 

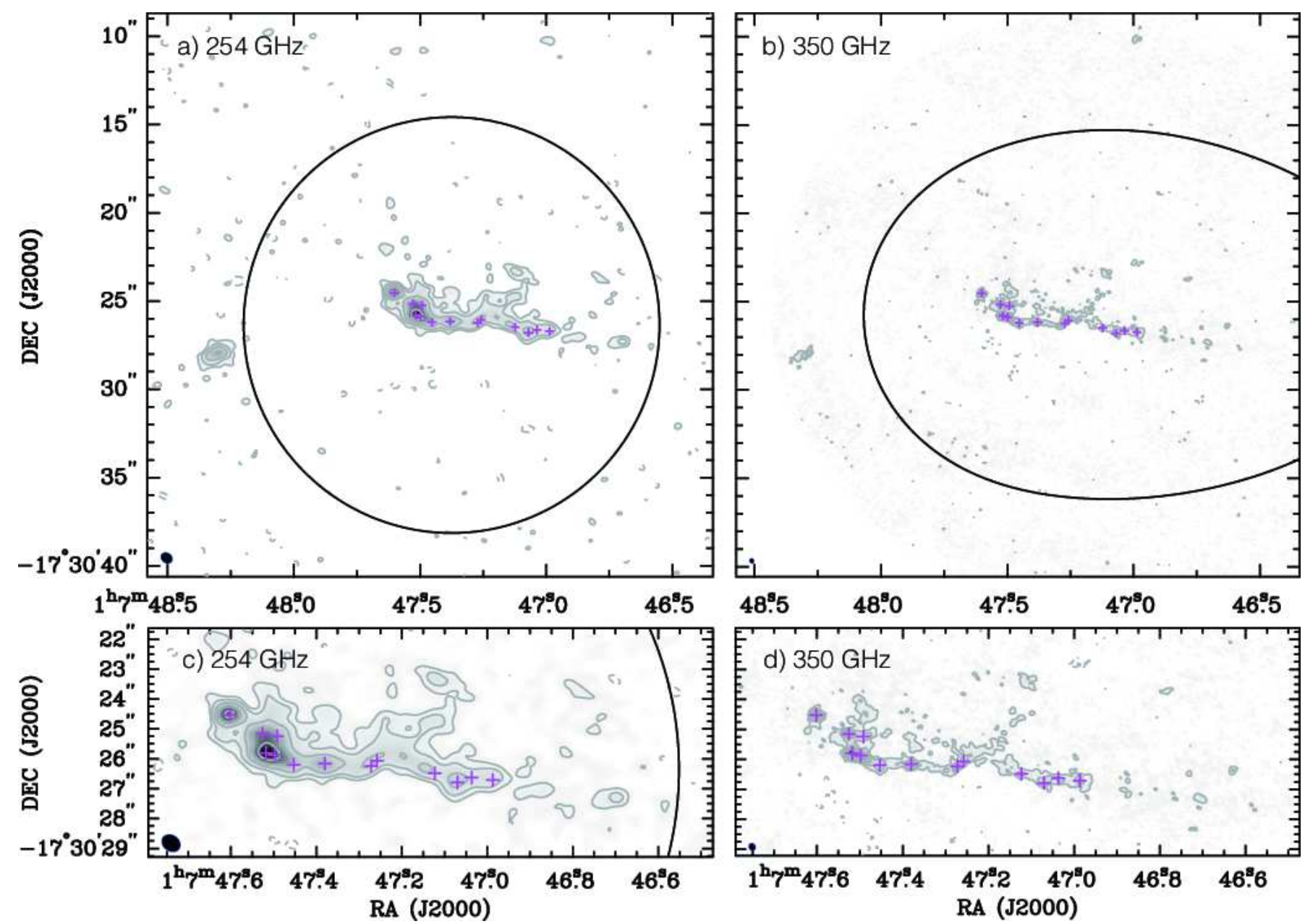

Figure 3. (a) The $254 \mathrm{GHz}$ continuum image of VV 114. The contours are $1 \sigma \times(-3,3,6,12,24,36$, and 48$) \mu \mathrm{Jy}^{\mathrm{b}}$ beam ${ }^{-1}$. $1 \sigma$ is 29 $\mu \mathrm{Jy}_{\text {beam }^{-1}}$. The black line shows the half power beam width (i.e., field of view). (b) The $350 \mathrm{GHz}$ continuum image of VV 114 . The contours are $1 \sigma \times(-3,3,6,12,24,36$, and 48$) \mu \mathrm{Jy}_{\text {beam }}{ }^{-1} .1 \sigma$ is $26 \mu \mathrm{Jy}_{\text {beam }}{ }^{-1}$. The black oval shape shows the half power beam width. Zoomed-in views of (a) and (b) for the filament are shown in (c) and (d), respectively. All 350 GHz peaks stronger than $8 \sigma$ are marked as crosses.

and dispersion maps are similar to those of the filament obtained in the $\mathrm{CO}(1-0)$ and ${ }^{13} \mathrm{CO}(1-0)$ lines (Saito et al. 2015). The largest velocity dispersion is found around the Overlap region, except for the HNC (1-0) and HCN (3-2) images. The widest velocity dispersion is found around the Overlap region in the HCN $(1-0)$, $\mathrm{HCO}^{+}(1-0)$, and $\mathrm{HCO}^{+}(3-2)$ images (Figures 4c, 4f, and $5 \mathrm{f})$. As clearly seen in the velocity field of $\mathrm{HCO}^{+}(3-$ 2) (Figure 5f), the wide dispersion $\left(>100 \mathrm{~km} \mathrm{~s}^{-1}\right)$ is explained by the superposition of the eastern redshifted component and the central blueshifted component (i.e., a double-peaked profile), not due to the intrinsic velocity dispersion of molecular gas clumps there. We use eleven $3^{\prime \prime} 0(\sim 1.2 \mathrm{kpc}$ in diameter) apertures along the filament of VV 114 as in Saito et al. (2017a) in order to measure the fluxes of the lines and continuum emission (See Figure $1 \mathrm{~b}$ inset), which are then used for the estimates of molecular gas surface density $\left(\Sigma_{\mathrm{H}_{2}}\right)$. The same apertures were adopted to estimate star formation rate surface densities $\left(\Sigma_{\mathrm{SFR}}\right)$ (Saito et al. 2017a). The measured fluxes are listed in Table 2.

\subsection{Clumpy Dense Gas Filament across VV 114}

The 80 pc resolution images of $\mathrm{HCN}(4-3)$ and $\mathrm{HCO}^{+}(4-3)$ emission (Figure 6) clearly show clumpy dense gas structures along the filament. The $\mathrm{HCO}^{+}$filament has a total length of $\sim 6 \mathrm{kpc}$ and a width of $\lesssim$ $200 \mathrm{pc}$, and consists of a dozen of giant molecular clouds (GMCs), most of which coincide with the $350 \mathrm{GHz}$ continuum peaks (Figures $3 \mathrm{~b}$ and $3 \mathrm{~d}$ ). Such morphological characteristics, together with the enhanced abundance of a molecular gas shock tracer, $\mathrm{CH}_{3} \mathrm{OH}$, found at the Overlap region (Saito et al. 2017a), share strong similarities with theoretical prediction that a colliding gas-rich galaxy pair forms a filamentary structure at the collision interface (i.e., shock front) between the progenitor's disks (e.g., Saitoh et al. 2009; Teyssier et al. 2010).

\subsection{The Eastern Nucleus}

We show zoom-up images of line and continuum emission around the eastern nucleus in Figure 7. Except for the coarse resolution Band 3 data, the nuclear region is resolved into multiple clumps. However, most clumps are not resolved even by the $80 \mathrm{pc}$ beam of the Band 7 data. All line peaks roughly coincide with the $350 \mathrm{GHz}$ continuum peaks, indicating that cold dust grains around 


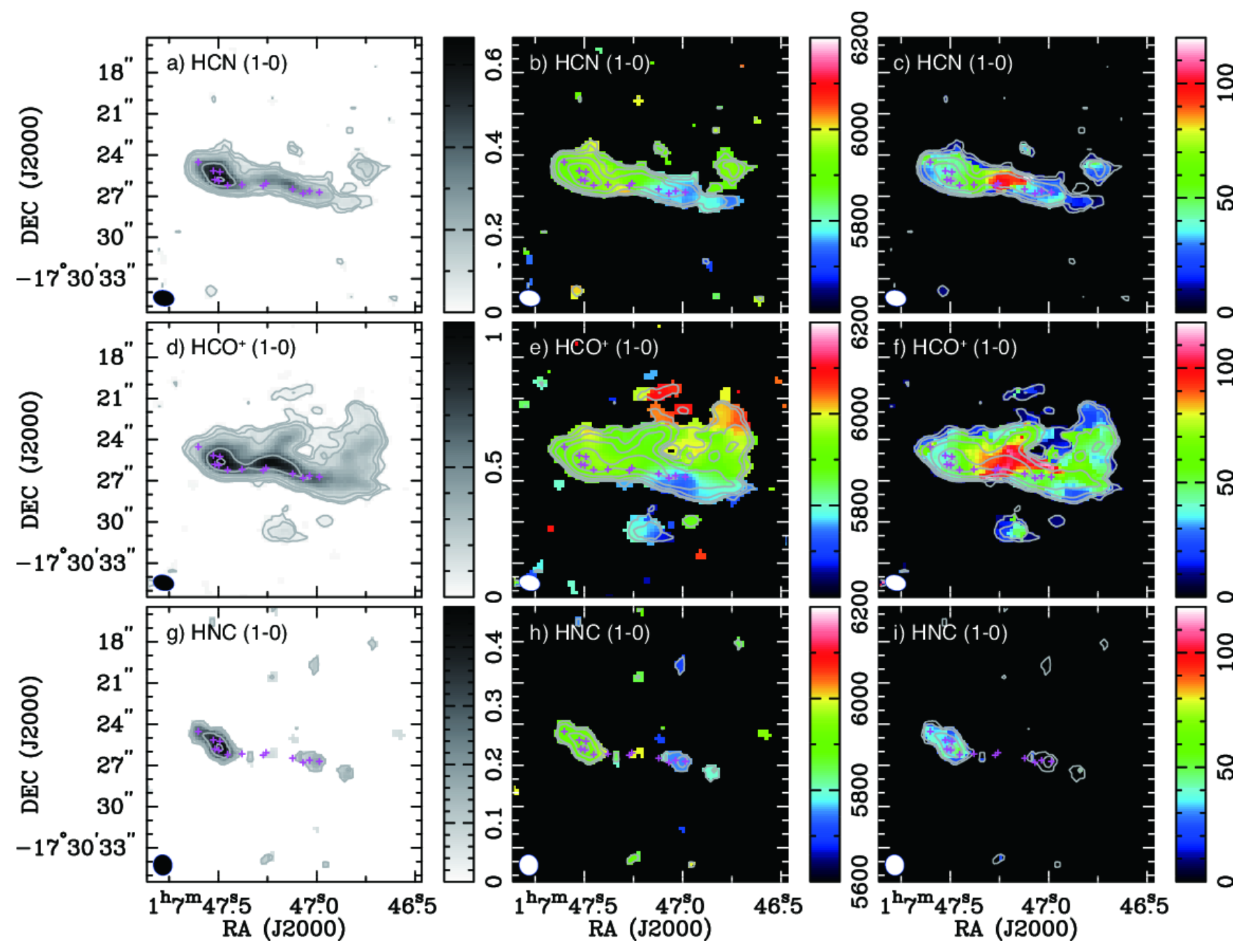

Figure 4. (a) Integrated intensity image of HCN (1-0) of VV 114 in units of Jy beam ${ }^{-1} \mathrm{~km} \mathrm{~s}^{-1}$. The synthesized beam is shown in the bottom left corner. The contours are $0.67 \times(0.04,0.08,0.16,0.32,0.64$, and 0.96$) \mathrm{Jy} \mathrm{beam}^{-1} \mathrm{~km} \mathrm{~s}^{-1}$. The magenta crosses correspond to the $350 \mathrm{GHz}$ peaks stronger than $8 \sigma\left(1 \sigma=26 \mu \mathrm{Jy}_{\text {beam }}^{-1}\right)$. (b) Velocity field image of HCN (1-0) of VV 114 in units of km s $\mathrm{s}^{-1}$ The integrated intensity image is shown as contours. (c) Velocity dispersion image of HCN (1-0) of VV 114 in units of km s $\mathrm{s}^{-1}$. The integrated intensity image is shown as contours. (d/e/f) Same as $(\mathrm{a} / \mathrm{b} / \mathrm{c})$ but for $\mathrm{HCO}^{+}(1-0)$. The contours are $1.06 \times(0.04,0.08,0.16,0.32,0.64$, and 0.96) $\mathrm{Jy} \mathrm{beam}^{-1} \mathrm{~km} \mathrm{~s}^{-1}$. (g/h/i) Same as $(\mathrm{a} / \mathrm{b} / \mathrm{c})$ but for HNC (1-0). The contours are $0.47 \times(0.16,0.32,0.64$, and 0.96$) \mathrm{Jy}$ beam ${ }^{-1}$ $\mathrm{km} \mathrm{s}^{-1}$.

the nuclear region are concomitant with dense molecular ISM with $80 \mathrm{pc}$ scale. In VV 114, the spatial coincidence between dense gas and dust emission is seen from kpc-scale to 80 pc-scale.

Although the Band 7 data provide the highest angular resolution image of molecular line toward VV 114 up to date, the $\mathrm{HCN}(4-3), \mathrm{HCO}^{+}(4-3)$, and $350 \mathrm{GHz}$ continuum images cannot resolve molecular gas structures around the putative AGN, which gives a size upper limit of $80 \mathrm{pc}$ in diameter. The AGN core also shows bright CS (7-6) emission (Figure 8), which was tentatively detected previously (Saito et al. 2015). Assuming that at the AGN position of all images there is a negligible flux contribution from surrounding star-forming clumps, we measure line and continuum fluxes for the easternmost $350 \mathrm{GHz}$ peak of the filament at $(\alpha, \delta)_{\mathrm{J} 2000}$ $=\left(01^{\mathrm{h}} 07^{\mathrm{m}} 47.60,-17^{\circ} 30^{\prime} 24^{\prime \prime} .52\right)$, and regard it as molecular ISM which might be dominantly affected by the puta- tive AGN of VV 114. We measured integrated fluxes at E0 (AGN), as well as E1 (a dense clump at $~ 11^{\prime \prime} 5$ southwest from E0), as defined by Iono et al. (2013), and listed them in Table 3. Fluxes at E1 might be strongly contaminated by other clumps in the Bands 3 and 6 images, so we only measure line and continuum fluxes in Band 7. Spectra of all bands toward the AGN position are shown in Figure 9. All lines show broad Gaussian-like profile (FWHM $\sim 200 \mathrm{~km} \mathrm{~s}^{-1}$ ), indicating a large dynamical mass of $M_{\text {dyn }}$ of $9.3 \times 10^{7} M_{\odot}$ assuming the inclination of $90^{\circ}$ for simplicity.

\subsection{Vibrationally-excited HCN Lines}

Our frequency coverage contains two vibrationallyexcited rotational transitions of $\mathrm{HCN}$, i.e., $J=3-2, v_{2}$ $=1^{1 f}$ at $\nu_{\text {rest }}=267.1993 \mathrm{GHz}\left(\nu_{\mathrm{obs}}=261.94 \mathrm{GHz}\right)$ and $J=4-3, v_{2}=1^{1 f}$ at $\nu_{\text {rest }}=356.2556 \mathrm{GHz}\left(\nu_{\mathrm{obs}}\right.$ $=349.25 \mathrm{GHz}$ ). Those vibrationally-excited HCN lines 

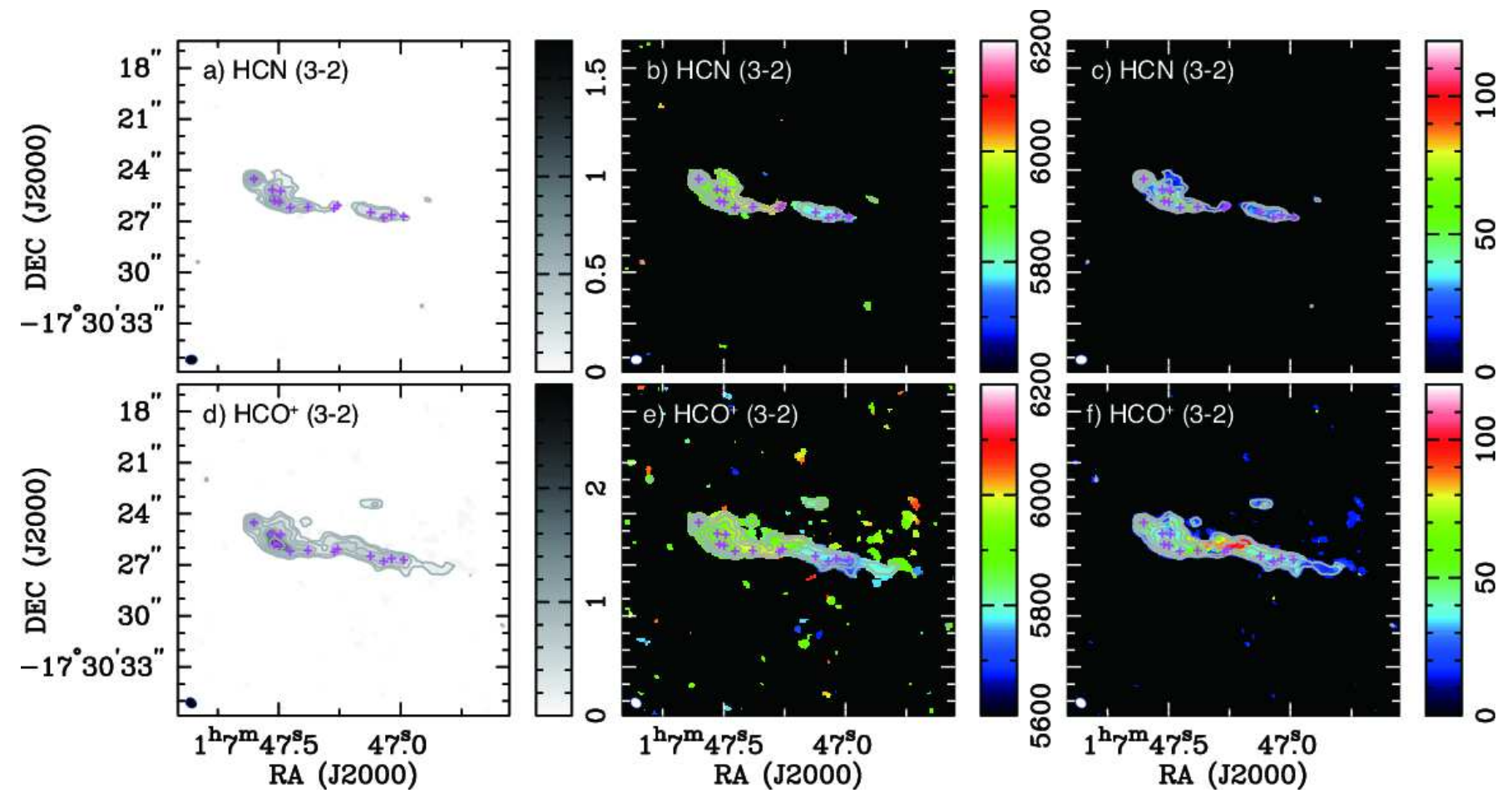

Figure 5. (a/b/c) Same as Figure 4a, b, and c, but for HCN (3-2). The contours are $1.69 \times(0.04,0.08,0.16,0.32,0.64$, and 0.96$)$ Jy beam ${ }^{-1} \mathrm{~km} \mathrm{~s}^{-1}$. The magenta crosses correspond to the $350 \mathrm{GHz}$ peaks stronger than $8 \sigma\left(1 \sigma=26 \mu \mathrm{Jy} \mathrm{beam}{ }^{-1}\right)$. (d/e/f) Same as Figure $4 \mathrm{a}, \mathrm{b}$, and $\mathrm{c}$, but for $\mathrm{HCO}^{+}(3-2)$. The contours are $2.91 \times(0.04,0.08,0.16,0.32,0.64$, and 0.96$) \mathrm{Jy} \mathrm{beam}^{-1} \mathrm{~km} \mathrm{~s}^{-1}$.
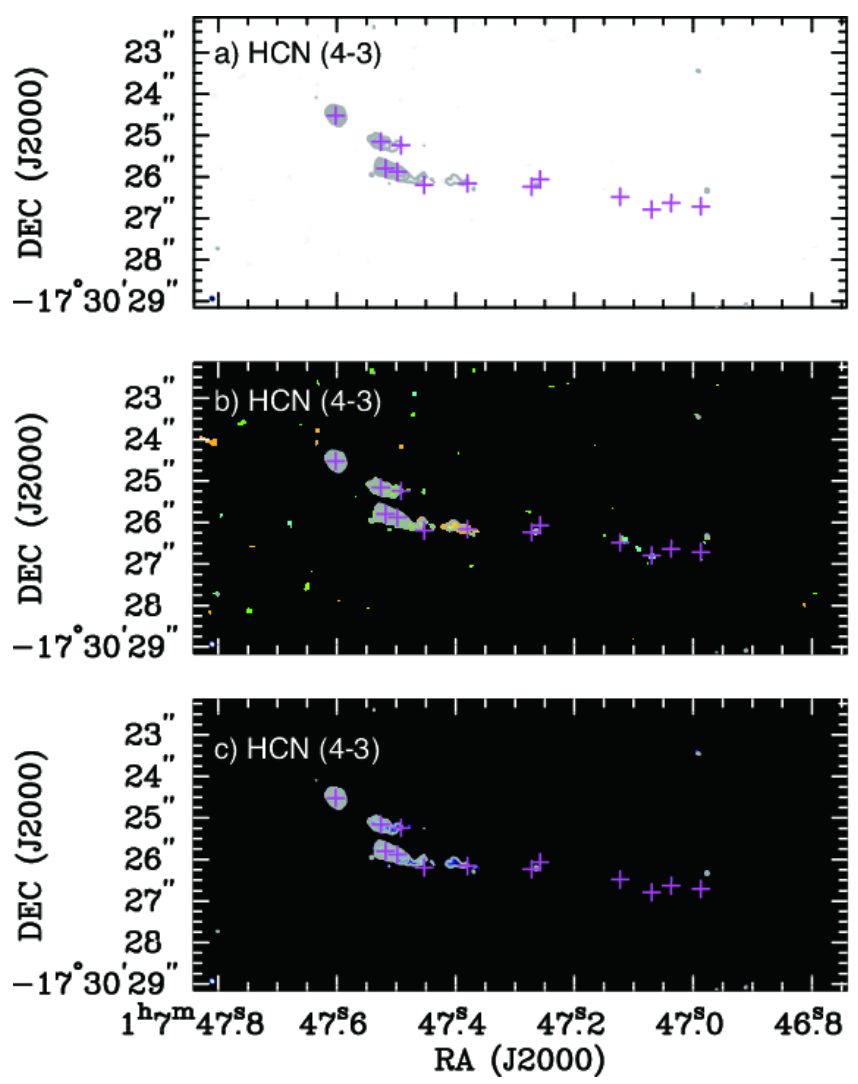
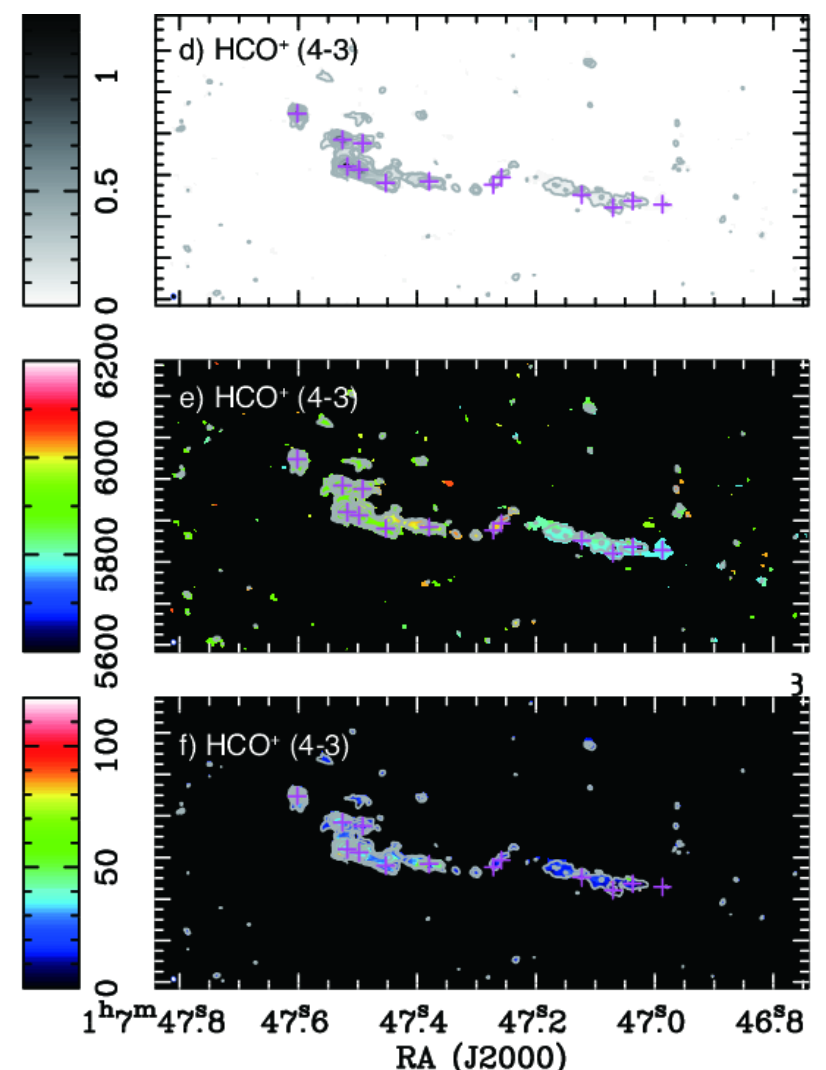

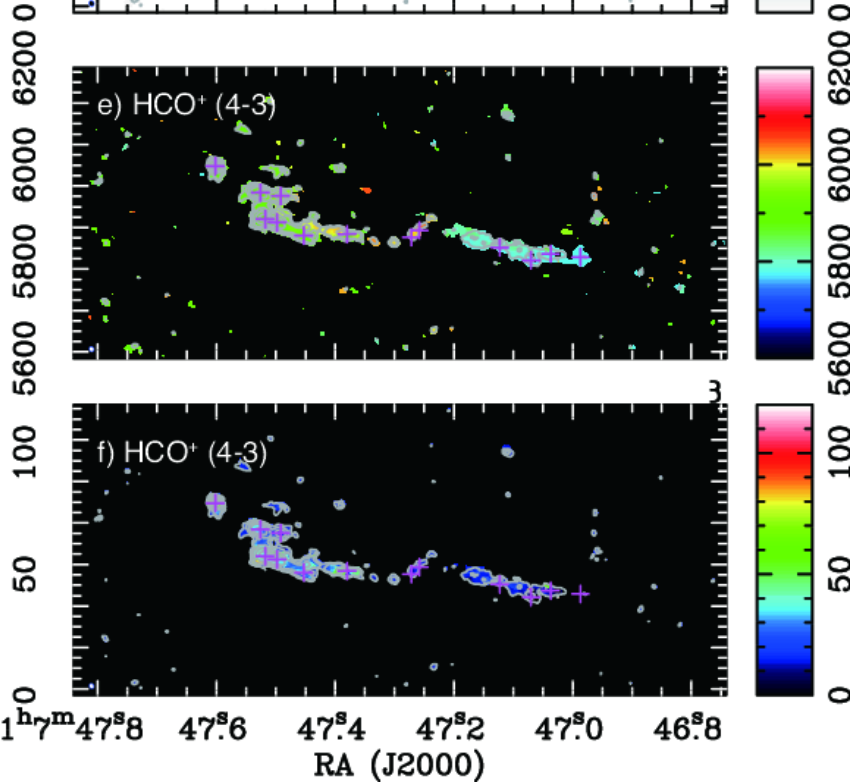

Figure 6. $(\mathrm{a} / \mathrm{b} / \mathrm{c})$ Same as Figure $4 \mathrm{a}, \mathrm{b}$, and c, but for HCN $(4-3)$. The contours are $1.27 \times(0.04,0.08,0.16,0.32,0.64$, and 0.96$)$ Jy beam ${ }^{-1} \mathrm{~km} \mathrm{~s}^{-1}$. The magenta crosses correspond to the $350 \mathrm{GHz}$ peaks stronger than $8 \sigma\left(1 \sigma=26 \mu \mathrm{Jy} \mathrm{beam}{ }^{-1}\right)$. (d/e/f) Same as Figure $4 \mathrm{a}, \mathrm{b}$, and c, but for $\mathrm{HCO}^{+}(4-3)$. The contours are $1.22 \times(0.04,0.08,0.16,0.32,0.64$, and 0.96$) \mathrm{Jy} \mathrm{beam}^{-1} \mathrm{~km} \mathrm{~s}^{-1}$. 

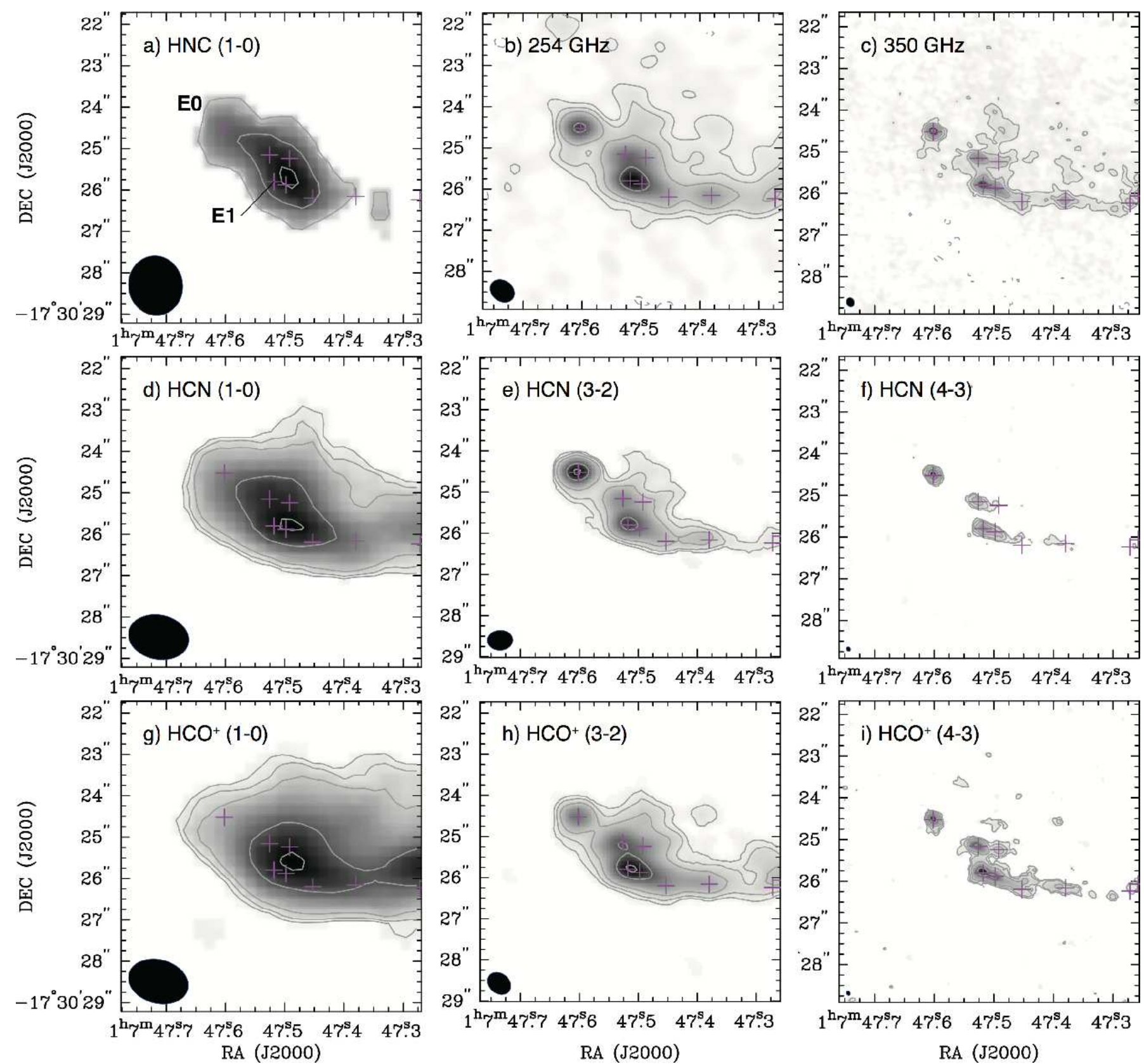

Figure 7. Zoomed-in integrated intensity images in the inner $7^{\prime \prime}$ of the eastern galaxy of (a) HNC (1-0), (b) $254 \mathrm{GHz}$ continuum, (c) $350 \mathrm{GHz}$ continuum, (d) HCN (1-0), (e) $\mathrm{HCN}(3-2)$, (f) $\mathrm{HCN}(4-3)$, (g) $\mathrm{HCO}^{+}(1-0)$, (h) $\mathrm{HCO}^{+}(3-2)$, and (i) $\mathrm{HCO}^{+}(4-3)$. The contours are the same as in previous figures. The magenta crosses correspond to the $350 \mathrm{GHz}$ peaks stronger than $8 \sigma\left(1 \sigma=26 \mu \mathrm{Jy}^{\mathrm{b}}\right.$ beam $\left.{ }^{-1}\right)$. 
ALMA Observations of $\mathrm{HCN}$ and $\mathrm{HCO}^{+}$SLEDs toward VV 114

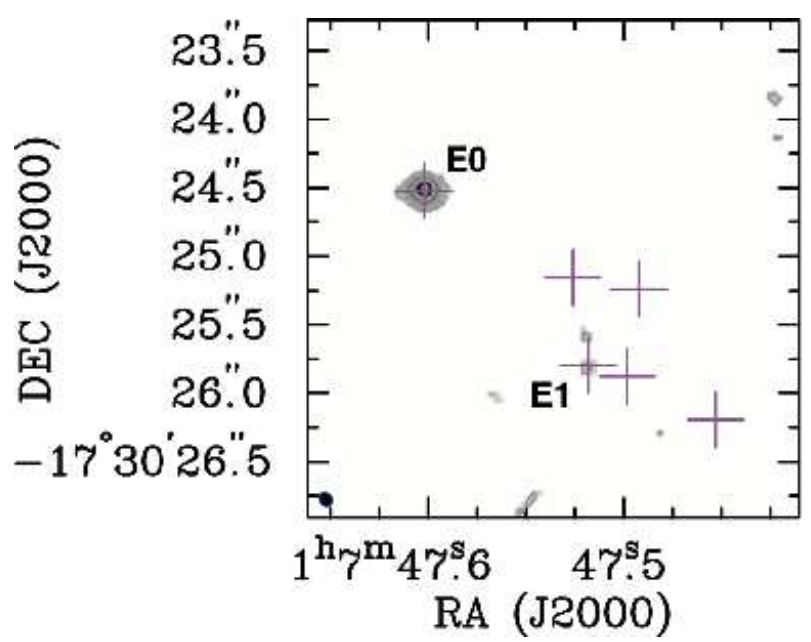

Figure 8. Zoomed-in integrated intensity image of CS (7-6). The contours are $0.26 \times(0.16,0.32,0.64$, and 0.96$) \mathrm{Jy} \mathrm{beam}^{-1} \mathrm{~km} \mathrm{~s}^{-1}$. The magenta crosses correspond to the $350 \mathrm{GHz}$ peaks stronger than $8 \sigma\left(1 \sigma=26 \mu \mathrm{Jy}^{\text {beam }^{-1}}\right)$.

have been frequently observed toward obscured nuclei in nearby (U)LIRGs (e.g., Sakamoto et al. 2010, 2013; Aalto et al. 2015b; Imanishi et al. 2016b, 2017), and thus they might be detectable toward the putative AGN in VV 114 (E0). Since the critical densities of those vibrationallyexcited $\mathrm{HCN}$ lines exceed $10^{10} \mathrm{~cm}^{-3}$, it is not easy to excite HCN molecules to a high vibrational states only by collisions. Instead, radiative excitation, in particular infrared radiation is required to explain detections of the vibrationally-excited HCN lines, and thus those lines are excellent tracers of hot, obscured nuclei in galaxies.

As shown in Figure 9, there is no statistically significant feature at these two frequencies. The $3 \sigma$ upper limit of $\operatorname{HCN}\left(J=3-2, v_{2}=1^{1 f}\right)$ and $\operatorname{HCN}\left(J=4-3, v_{2}=\right.$ $1^{1 f}$ ) are $0.26 \mathrm{Jy} \mathrm{km} \mathrm{s}^{-1}$ and $0.26 \mathrm{Jy} \mathrm{km} \mathrm{s}^{-1}$, respectively, assuming $\mathrm{FWHM}=200 \mathrm{~km} \mathrm{~s}^{-1}$, which is the observed linewidth of HCN (4-3) and HCN (3-2) at E0. The observed flux ratios between $v_{2}=1^{1 f}$ and $v_{2}=0$ are then $<0.15$ for $J=3-2$ and $<0.16$ for $J=4-3$. If compared with the deeply obscured LIRG NGC 4418, which shows 0.17 and 0.23 for $J=3-2$ and $J=4-3$, respectively (Sakamoto et al. 2010), the AGN in VV 114 has weaker $v_{2}=1^{1 f}$ fluxes normalized by $v_{2}=0$ fluxes.

\section{LINE RATIOS}

In this Section, we used the $u v$-clipped and beamconvolved images to construct line ratio images.

\subsection{Excitation Ratios}

The excitation of dense molecular gas in galaxies has been investigated for local galaxies, bright high-z systems, and, in particular, their bright nuclei (Krips et al. 2008; Izumi et al. 2013; Papadopoulos et al. 2014; Spilker et al. 2014; Tunnard et al. 2015), although its spatial variation in LIRGs has not been studied in detail due to the faintness of dense gas tracers. Here we show spatially-resolved HCN $\left(J_{\mathrm{upp}}-J_{\mathrm{upp}-1}\right) / \mathrm{HCN}(1-0)$ line ratios and $\mathrm{HCO}^{+}\left(J_{\mathrm{upp}}-J_{\mathrm{upp}-1}\right) / \mathrm{HCO}^{+}(1-0)$ line ratios (hereafter $\mathrm{HCN}$ or $\mathrm{HCO}^{+}$excitation ratios) for a LIRG for the first time (Figure 10). All excitation ratios are made before clipping the inner $u v$-coverage (see
Section 2.4) and convolving to $1^{\prime \prime} .5$ resolution $(\sim 600 \mathrm{pc})$. The $\mathrm{HCN}\left(\mathrm{HCO}^{+}\right)$excitation ratios are masked to the region delimited by the $\mathrm{HCN}(1-0)\left(\mathrm{HCO}^{+}(1-0)\right)$ integrated intensity level at $0.095(0.153) \mathrm{Jy} \mathrm{beam}^{-1} \mathrm{~km}$ $\mathrm{s}^{-1}$ because it corresponds to $3 \sigma$ levels in the convolved integrated intensity maps.

The overall spatial distributions of the $\mathrm{HCN}$ and $\mathrm{HCO}^{+}$excitation ratios in VV 114 are coincident with each other. In all cases, the peak is located at the eastern part of the filament. All excitation ratios do not exceed the optically-thick thermalized value (unity when using $\mathrm{K} \mathrm{km} \mathrm{s}^{-1}$ units) anywhere. The highly-excited region at the eastern nucleus extends from northeast to southwest, and coincides with the HNC (1-0) distribution (Figure 7a). The Overlap region is also relatively excited, but a few times lower than the ratios around the eastern nucleus. The excitation of diffuse molecular gas phase traced by lower- $J \mathrm{CO}$ or ${ }^{13} \mathrm{CO}$ excitation ratios (Sliwa et al. 2013; Saito et al. 2015) shows a similar distribution along the filament with a peak at the eastern edge, indicating that the heating source of the diffuse gas is similar to that of the dense gas.

\section{2. $\mathrm{HCN} / \mathrm{HCO}^{+}$Ratios}

Contrary to the excitation ratios, intensity ratios between $\mathrm{HCN}$ and $\mathrm{HCO}^{+}$for a given $J$ have been studied to characterize obscured nuclear regions (i.e., AGN or starburst), although $\mathrm{HCN} / \mathrm{HCO}^{+}$ratios at multiple $J$ are not broadly studied, except for some nearby bright galaxies (Krips et al. 2008; Izumi et al. 2013), because of the line weakness and the instrumental limitations. Using the high quality data of multiple $J \mathrm{HCN}$ and $\mathrm{HCO}^{+}$ transitions taken for $\mathrm{VV} 114$, we made $\mathrm{HCN} / \mathrm{HCO}^{+}$ratio images at $J=1-0,3-2$, and $4-3$ as shown in Figures 11a, 11b, and 11c, respectively. Here we used integrated intensity images without correcting the primary beam attenuation, smoothing the synthesized beam, and $u v$-clipping, because the antenna configurations are virtually identical for $\mathrm{HCN}$ and $\mathrm{HCO}^{+}$at a given $J$ (Table 1).

All $\mathrm{HCN} / \mathrm{HCO}^{+}$ratio images show the highest peak $(\sim 1.5)$ at the putative AGN position (E0) despite of the different resolutions. The second peak is located at $\sim 1$.'5 southwest of the AGN position, which coincides with a strong peak found in all images shown in Figure 7 (i.e., star-forming dense clumps). The second peak value varies from 0.7 to 0.45 as $J$ increases. The Overlap region shows relatively high values of $\sim 0.4$ independently of $J$. In terms of multiple $J \mathrm{HCN}^{2}$ and $\mathrm{HCO}^{+}$ line ratios, VV 114, therefore, has three distinctive regions, AGN, star-forming clump(s), and Overlap region, which in turn shows the kpc-scale variation of dense gas excitation and chemistry along the filament. The higher $\mathrm{HCN} / \mathrm{HCO}^{+}$ratios only near the putative AGN position is consistent with previous studies (García-Burillo et al. 2014; Viti et al. 2014; Imanishi et al. 2016c, and references therein). Note that recent high angular resolution observations of $\mathrm{HCN}$ and $\mathrm{HCO}^{+}$lines revealed (e.g., Martín et al. 2015; Espada et al. 2017; Salak et al. 2018) that the $\mathrm{HCN} / \mathrm{HCO}^{+}$ratios show a variety of spatial variations within circumnuclear disks.

\section{3. $H N C / H C N$ and $H C N / C S$ Ratio}




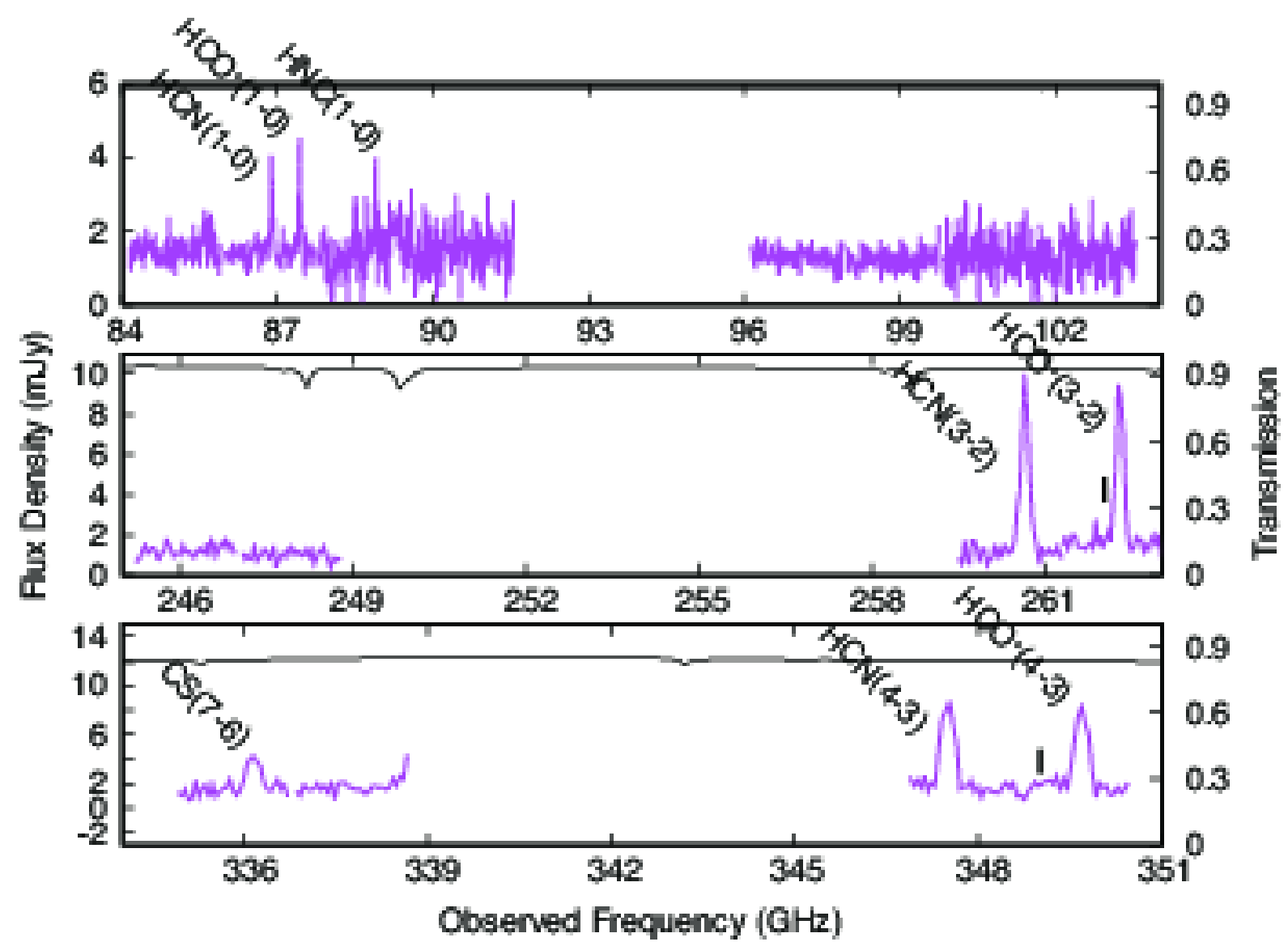

Figure 9. Spectra (line + continuum) toward the putative AGN position (E0) at all bands. The velocity resolution is $50 \mathrm{~km} \mathrm{~s}^{-1}$. Atmospheric transmission with precipitable water vapor of $1 \mathrm{~mm}$ is shown in black lines. The two vertical lines at the left side of $\mathrm{HCO}^{+}(3-2)$ and $\mathrm{HCO}^{+}(4-3)$ show the observed frequency of $\mathrm{HCN}\left(v_{2}=1^{1 f}, 3-2\right)$ and $\mathrm{HCN}\left(v_{2}=1^{1 f}, 4-3\right)$, respectively

We detected HNC (1-0) and CS (7-6) lines toward the AGN position. The $\mathrm{HNC}(1-0) / \mathrm{HCN}(1-0)$ line ratio at $\mathrm{E} 0$ is $0.85 \pm 0.23$, indicating a comparable strength of $\mathrm{HNC}$ to $\mathrm{HCN}$ at the AGN position. When using $33^{\prime \prime} 0$ apertures (Figure 1b), Region 3 shows an HNC (1$0) / \mathrm{HCN}(1-0)$ ratio of $\sim 0.25$ ( $<0.4$ for other positions). The AGN position shows significantly brighter HNC luminosity relative to other molecular regions in VV 114. $\mathrm{HNC} / \mathrm{HCN} \gtrsim 1$ is seen in active nuclei (e.g., Huettemeister et al. 1995; Aalto et al. 2002; Pérez-Beaupuits et al. 2007; Costagliola et al. 2011; Aladro et al. 2015, see also Aalto et al. 2007 for $J=3-2$ transition). HCN (43)/CS (7-6) line ratio is suggested to be a diagnostic tracer of AGN activity when combining with the $\mathrm{HCN}(4-3) / \mathrm{HCO}^{+}(4-3)$ line ratio (e.g. Izumi et al. 2016). The HCN (4-3)/CS (7-6) ratio is $4.9 \pm 1.3$ at the AGN position of VV 114 (E0). The AGN may have a lower ratio than those in the other regions because it is similar to the lowest $3 \sigma$ lower limit $(>5.2)$ of all the other apertures. On the submillimeter-HCN diagram using $\mathrm{HCN}(4-3) / \mathrm{HCO}^{+}(4-3)$ and $\mathrm{HCN}(4-3) / \mathrm{CS}(7-6)$ ratios (Izumi et al. 2016), E0 is similar to NGC 4418 (Sakamoto et al. 2013) and IRAS12127-1412 (Imanishi \& Nakanishi 2014), both of which may harbor a dustobscured AGN.
Recent observations have revealed a variety of excitation conditions in dense gas ISM from galaxy to galaxy by comparing $\mathrm{HCN}$ and $\mathrm{HCO}^{+}$spectral line energy distribution (SLED) (e.g., Rangwala et al. 2011; Papadopoulos et al. 2014). In Figure 12a and 12b we show spatiallyresolved $\mathrm{HCN}$ and $\mathrm{HCO}^{+}$SLEDs, respectively, for the first time in this source, using the eleven apertures (Regions 1-11) and data at the AGN position (E0). The $\mathrm{HCO}^{+}$SLEDs of VV 114, in general, tend to show larger values relative to the HCN SLEDs at a given $J$. Both SLEDs for Region 1-3 and E0 peak at $J \gtrsim 3$, whereas those for Region 4-11 (i.e., Overlap) peak at $J \lesssim 3$. On the other hand, Region 1 (and E0) only show high values $(\gtrsim 1)$ in $\mathrm{HCN} / \mathrm{HCO}^{+}$ratios (Figure $12 \mathrm{c}$ ). The shape of the $\mathrm{HCN} / \mathrm{HCO}^{+}$ratio with $J$ (hereafter $\mathrm{HCN} / \mathrm{HCO}^{+}$ ratio SLED) at E0 is remarkably flat and higher than unity, indicating a specific gas condition relative to other regions. Since those spatially-resolved SLEDs depend on the variety of thermal and chemical processes in ISM along the filament, we model those in Section 5.5 to discuss driving mechanisms.

\section{DISCUSSION}

5.1. $L_{\mathrm{FIR}}-L_{\mathrm{dense}}^{\prime}$ Relation and Slope - J Dependence

Our $\mathrm{HCN}$ and $\mathrm{HCO}^{+}$data have a similar beam size for a given $J$, sensitivity, and maximum recoverable scale, allowing us to probe the dense gas - star-forming scaling 


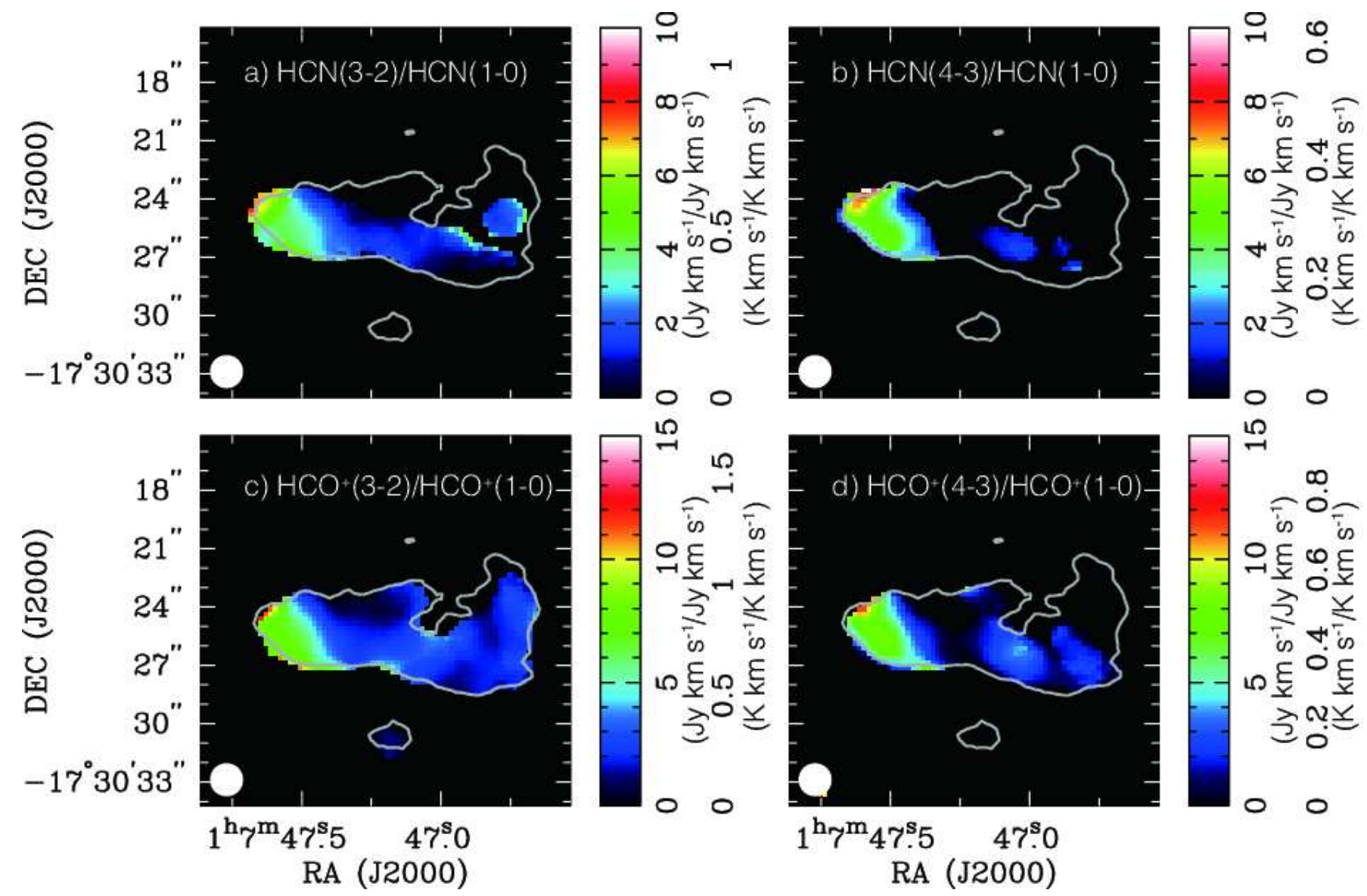

Figure 10. (a) HCN (3-2)/HCN (1-0) integrated intensity ratio map. The $\mathrm{HCO}^{+}(1-0)$ contour $\left(=0.0766 \mathrm{Jy} \mathrm{beam}^{-1} \mathrm{~km} \mathrm{~s}^{-1}\right)$ is shown as a grey contour. This contour is the same as the first contour of Figure 4d. (b) HCN (4-3)/HCN (1-0) integrated intensity ratio map. (c) $\mathrm{HCO}^{+}(3-2) / \mathrm{HCO}^{+}$(1-0) integrated intensity ratio map. (d) $\mathrm{HCO}^{+}(4-3) / \mathrm{HCO}^{+}$(1-0) integrated intensity ratio map. Angular resolution is indicated in the bottom-left of each map.

relation without systematic errors to derive fluxes of the dense gas tracers. In this Paper, we use the SFR surface density $\left(\Sigma_{\mathrm{SFR}}\right)$ based on $110 \mathrm{GHz}$ continuum emission (see Saito et al. 2017a), and $\mathrm{HCN}$ and $\mathrm{HCO}^{+}$flux densities as a proxy of $L_{\mathrm{FIR}}$ and $L_{\text {dense }}^{\prime}$, respectively. Here we assumed that free-free (bremsstrahlung) emission dominates the $110 \mathrm{GHz}$ continuum emission, and used the free-free continuum to SFR conversion prescription described in Yun \& Carilli (2002). We note that the derived SFR is upper limit for E0 and Regions 1 and 2, because there might be a contribution from the nonthermal synchrotron emission from the putative AGN.

We roughly estimated the possible synchrotron contribution to the $110 \mathrm{GHz}$ flux at the Overlap region using measured $8.44 \mathrm{GHz}$ and $110 \mathrm{GHz}$ fluxes (Saito et al. 2015). Assuming that the spectral index of the synchrotron emission is -0.80 and that the synchrotron dominates $80 \%$ of the $8.44 \mathrm{GHz}$ flux (similar to M82; Condon 1992), the synchrotron contribution to the $110 \mathrm{GHz}$ flux is calculated to be $\sim 25 \%$. This is not a small value but it does not change any results and discussion in this Paper, because the apertures around the Overlap region also present a similar value of $\sim 25 \%$. We need further data points between radio and FIR to precisely evaluate thermal and nonthermal fluxes, which is beyond the scope of this Paper. We further discuss the slope of $\Sigma_{\mathrm{SFR}}$ and flux density relations here. The ratio (i.e., the star for- mation efficiency of dense gas, $\left.\mathrm{SFE}_{\text {dense }}=\mathrm{SFR} / M_{\text {dense }}\right)$ is discussed in Section 5.2.

In Figure $13, \Sigma_{\mathrm{SFR}}$ is plotted against $\mathrm{HCN}$ and $\mathrm{HCO}^{+}$ integrated fluxes. In general, integrated flux densities roughly correlate with $\Sigma_{\mathrm{SFR}}$ in log scale as pointed out in many studies (e.g., Gao \& Solomon 2004a,b; Privon et al. 2015; Usero et al. 2015; Shimajiri et al. 2017). We estimate the slope by fitting all the data points except Region 1, where considerable contamination by the putative AGN might be possible. The derived slope for $\Sigma_{\mathrm{SFR}}$ vs. $\mathrm{HCN}(1-0), \mathrm{HCN}(3-2), \mathrm{HCN}(4-3), \mathrm{HCO}^{+}(1-0)$, $\mathrm{HCO}^{+}(3-2)$, and $\mathrm{HCO}^{+}(4-3)$ fluxes are $2.03 \pm 0.18$, $0.83 \pm 0.07,0.65 \pm 0.04,1.69 \pm 0.67,1.30 \pm 0.16$, and $0.84 \pm 0.14$, respectively. Although both the number of data points and the covered $\mathrm{x}$ - and $\mathrm{y}$-ranges are small, the slope vary for different dense gas tracers. To clarify the excitation dependence, we plot the slope as a function of upper- $J$ in Figure 14, showing a decreasing trend as upper- $J$ increases of both $\mathrm{HCN}$ and $\mathrm{HCO}^{+}$(from $\sim 2$ to $\sim 0.5)$. This can be explained by two simple mechanisms, as we discuss next:

1. At the lower $\Sigma_{\mathrm{SFR}}$ regime $\left(\lesssim 20 M_{\odot} \mathrm{yr}^{-1} \mathrm{kpc}^{-2}\right)$, FUV heating by star-forming activity is inefficient and not enough to thermalize high critical density tracers toward higher- $J$. Consequently, flux densities of $J=3-2$ and 4-3 lines of $\mathrm{HCN}$ and $\mathrm{HCO}^{+}$ 


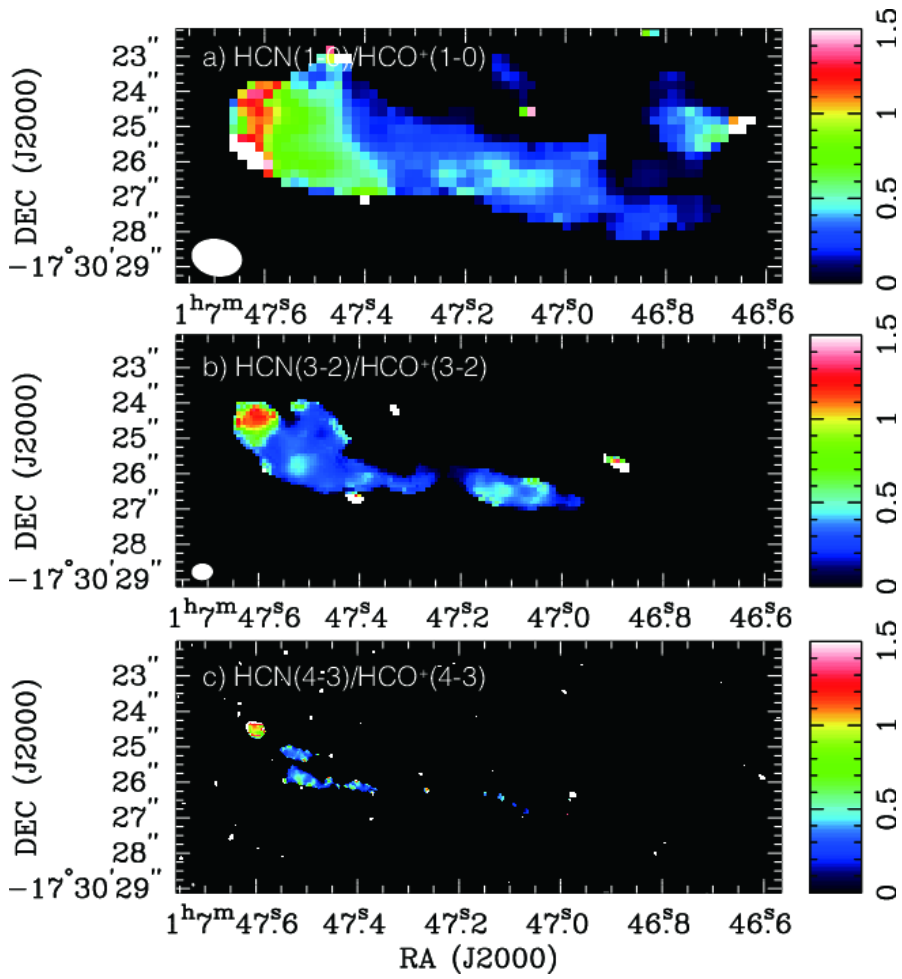

Figure 11. (a) Image of $\mathrm{HCN}(1-0) / \mathrm{HCO}^{+}(1-0)$ integrated in-

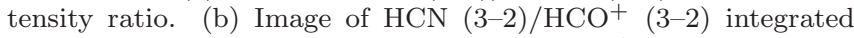
intensity ratio. (c) Image of $\mathrm{HCN}(4-3) / \mathrm{HCO}^{+}(4-3)$ integrated intensity ratio.

decrease, and thus the index becomes sublinear. Hydrodynamic simulations with non-LTE radiative transfer calculations (Narayanan et al. 2008) reproduced this trend for HCN transitions from $J=1-0$ to 5-4. An analytic model provided by Krumholz \& Thompson (2007) also reproduced this similar trend as a function of critical density.

2. At the higher $\Sigma_{\mathrm{SFR}}$ regime $\left(\gtrsim 20 M_{\odot} \mathrm{yr}^{-1} \mathrm{kpc}^{-2}\right)$, high critical density tracers can be easily excited by intense FUV radiation from massive star-forming regions (i.e., efficient collisional excitation due to high temperature or density). Furthermore, the presence of energetic activities (e.g., shock, AGN) tends to excite molecular gas ISM. Recent multiple $J$ CO observations have revealed that higher $J$ CO lines are more dominantly heated by such energetic activities (Greve et al. 2014), and brighter galaxies at IR show more excited CO conditions (Kamenetzky et al. 2016). Expanding those evidences to dense gas tracers, excitation of dense gas ISM at higher $\Sigma_{\text {SFR }}$ regime (i.e., higher IR) should be enhanced by energetic activities. Thus, the presence of intense starbursts and energetic activities can boost fluxes of higher $J \mathrm{HCN}$ and $\mathrm{HCO}^{+}$lines, leading to the sublinear slope. This is consistent with the explanation of a decreasing index with increasing $J$ of $\mathrm{CO}$ due to an increasing contribution of mechanical heating from supernovae (Greve et al. 2014). We will quantitatively discuss the excitation mechanisms of the dense gas tracers in Section 5.5.
Considering the observational fact that AGN contribution to the total IR luminosity increases as the IR luminosity increases (e.g., Ichikawa et al. 2014), the presence of AGN can boost higher $J \mathrm{HCN}$ and $\mathrm{HCO}^{+}$fluxes. However, we exclude the data point which might be dominated by AGN (i.e., Region 1) when deriving the slope, so this is not an applicable explanation for the case of the filament of VV 114.

We note that the $\Sigma_{\mathrm{SFR}}$ - integrated flux plots are equivalent to the FIR luminosity - dense gas luminosity relations in the sense that all data points shown here can be assumed to have a similar distance, aperture size, and filling factor, and thus, the slope will not change.

$$
\text { 5.2. } S F E_{\mathrm{H}_{2}}, S F E_{\text {dense, }} \text { and } f_{\text {dense }}
$$

Here we discuss the star formation activity which takes place in the filament of VV 114 using $\Sigma_{\mathrm{SFR}}$, molecular gas mass surface densities $\left(\Sigma_{\mathrm{H}_{2}}\right)$, and dense gas mass surface densities $\left(\Sigma_{\text {dense }}\right)$. In addition, we obtain $\mathrm{SFE}_{\mathrm{H}_{2}}$ $\left(=\Sigma_{\mathrm{SFR}} / \Sigma_{\mathrm{H}_{2}}\right), \mathrm{SFE}_{\text {dense }}\left(=\Sigma_{\mathrm{SFR}} / \Sigma_{\text {dense }}\right)$, and dense gas fraction, $\mathrm{f}_{\text {dense, }}\left(=\Sigma_{\text {dense }} / \Sigma_{\mathrm{H}_{2}}\right)$. We use $\Sigma_{\mathrm{H}_{2}}$ as in Saito et al. (2017a), which utilizes the $880 \mu \mathrm{m}$ dust continuum emission and the formulation to obtain molecular gas mass from the $880 \mu \mathrm{m}$ flux density described in Scoville et al. (2016). $\Sigma_{\text {dense }}$ is calculated by dividing the dense gas mass $\left(M_{\text {dense }}\right)$ by the aperture area. $M_{\text {dense }}$ is calculated using the following equation (Gao \& Solomon 2004b),

$$
M_{\text {dense }}=\alpha_{\mathrm{HCN}(1-0)} L_{\mathrm{HCN}(1-0)}^{\prime} M_{\odot}
$$

where $\alpha_{\mathrm{HCN}(1-0)}$ is the $\mathrm{HCN}(1-0)$ luminosity to dense gas mass conversion factor in $M_{\odot}\left(\mathrm{K} \mathrm{km} \mathrm{s}^{-1} \mathrm{pc}^{2}\right)^{-1}$ and $L_{\mathrm{HCN}(1-0)}^{\prime}$ is the $\mathrm{HCN}(1-0)$ luminosity in $\mathrm{K} \mathrm{km} \mathrm{s}^{-1} \mathrm{pc}^{2}$. We adopted $10 M_{\odot}\left(\mathrm{K} \mathrm{km} \mathrm{s}^{-1} \mathrm{pc}^{2}\right)^{-1}$ for $\alpha_{\mathrm{HCN}(1-0)}$ as in Gao \& Solomon (2004b). The derived surface densities are listed in Table 4.

We show surface densities along the filament of VV 114 in Figure 15a. To compare the overall trends between three quantities, we normalized all data points by their peak values. The normalization of $\Sigma_{\mathrm{SFR}}, \Sigma_{\mathrm{H}_{2}}$, and $\Sigma_{\text {dense }}$ is $56.3 M_{\odot} \mathrm{kpc}^{-2} \mathrm{yr}^{-1}, 1420 M_{\odot} \mathrm{pc}^{-2}$, and $268 M_{\odot}$ $\mathrm{pc}^{-2}$, respectively. All profiles peak close to the eastern nucleus (Region 2 or 3 ), and show a decreasing trend toward the western side (Region 11). This indicates that Region 2 and 3 are sites of ongoing massive, intense star formation (except for the AGN position, region 1). On the other hand, the Overlap region (Region 5-8) show moderate star formation ( $\sim 1 / 5$ of the nuclear star formation) with relatively large amount of diffuse and dense gas $(\sim 1 / 2$ of gas mass or surface density around the eastern nucleus). This trend becomes clearer when we use the surface density ratios (i.e., $\mathrm{SFE}_{\mathrm{H}_{2}}, \mathrm{SFE}_{\text {dense, }}$, and $f_{\text {dense }}$ ). These are listed in Table 4, and shown in Figure 15b. Note that both SFEs correspond to the intercept of the $\log L_{\mathrm{IR}}-\log L_{\text {line }}^{\prime}$ relations with a slope of unity. SFEs are high around the eastern nucleus (2-3 times higher than those at the Overlap region). The western side of the filament (Region 11) also shows high $\mathrm{SFE}_{\mathrm{H}_{2}}$, which is comparable to that at Region 2 and 3 . Region 11 coincides with strong $\mathrm{H} \alpha$ (Zaragoza-Cardiel et al. 2016) and $\mathrm{Pa} \alpha$ (Tateuchi et al. 2015) peaks, so this may be a relatively unobscured (i.e., not dusty) star-forming region associated with the western galaxy (Grimes et al. 

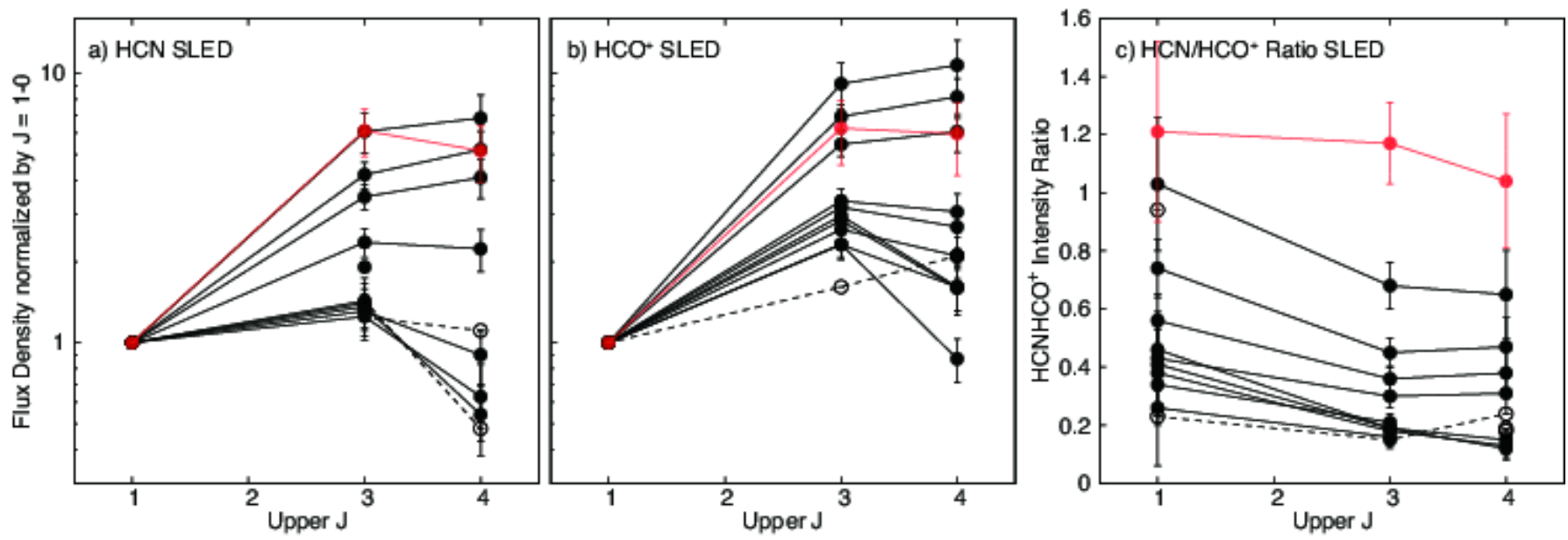

Figure 12. The spatially-resolved SLEDs of (a) $\mathrm{HCN}$, (b) $\mathrm{HCO}^{+}$, and (c) $\mathrm{HCN} / \mathrm{HCO}^{+}$ratio. Black and red lines correspond to data for Region 1-11 and for the AGN position, respectively. The open circles and dashed lines show $3 \sigma$ upper limits. Note that the upper three black tracks in all figures correspond to Region 1-3 (i.e., easternmost three apertures).
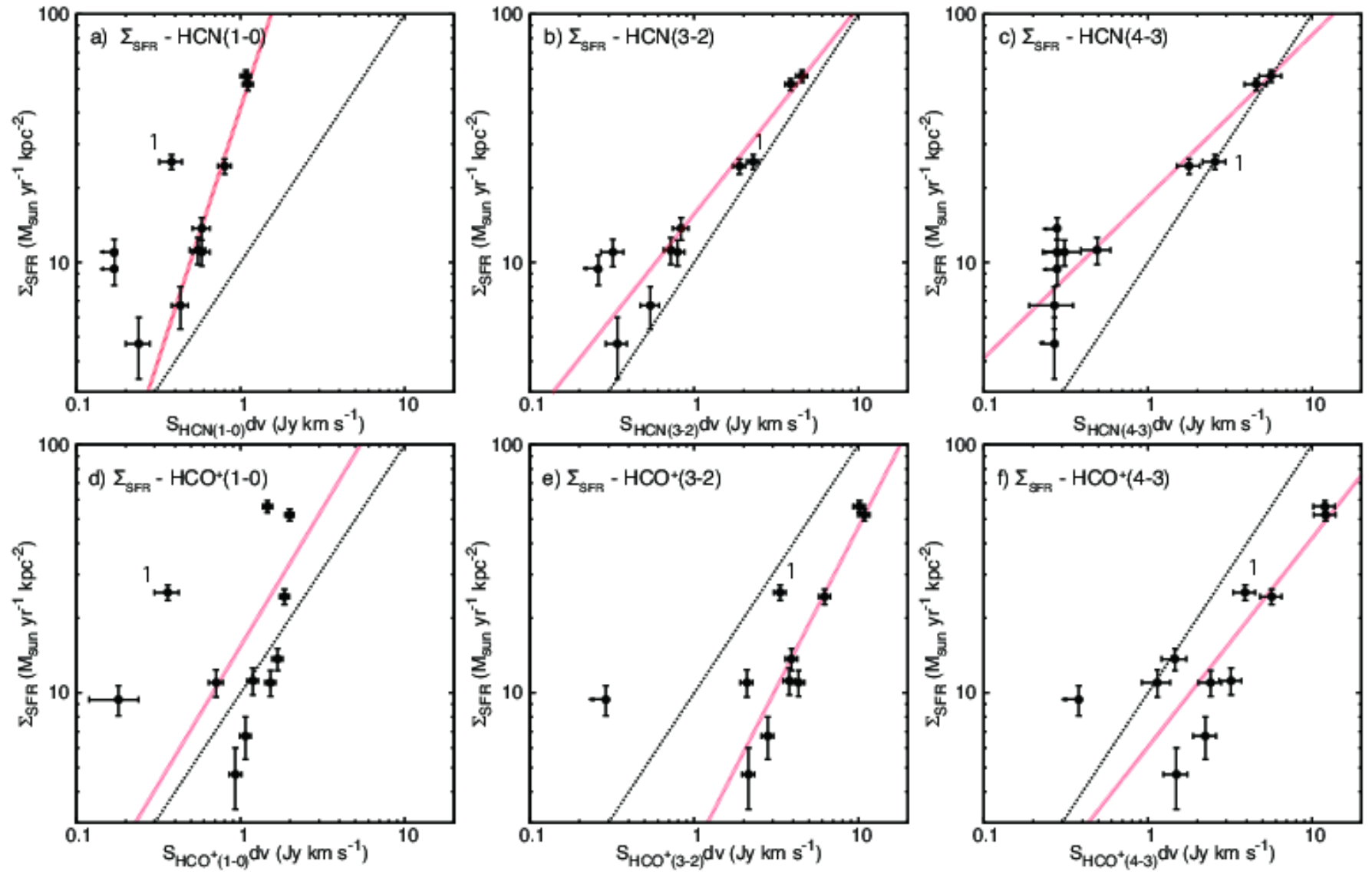

Figure 13. Plots of $\Sigma_{\mathrm{SFR}}$ against integrated fluxes of all $\mathrm{HCN}$ and $\mathrm{HCO}^{+}$transitions in $\log$ scale for the $3^{\prime \prime}$ apertures along the gaseous filament of VV 114 (see the inset of Figure 1b). These are the same as $\log L_{\text {dense }}^{\prime}-\log L_{\mathrm{IR}}$ plots. The black dotted line corresponds to a line with a slope of unity. The data point corresponding to Region 1 is denoted as 1 . The best fitted lines excluding Region 1 are shown as red dotted lines. 


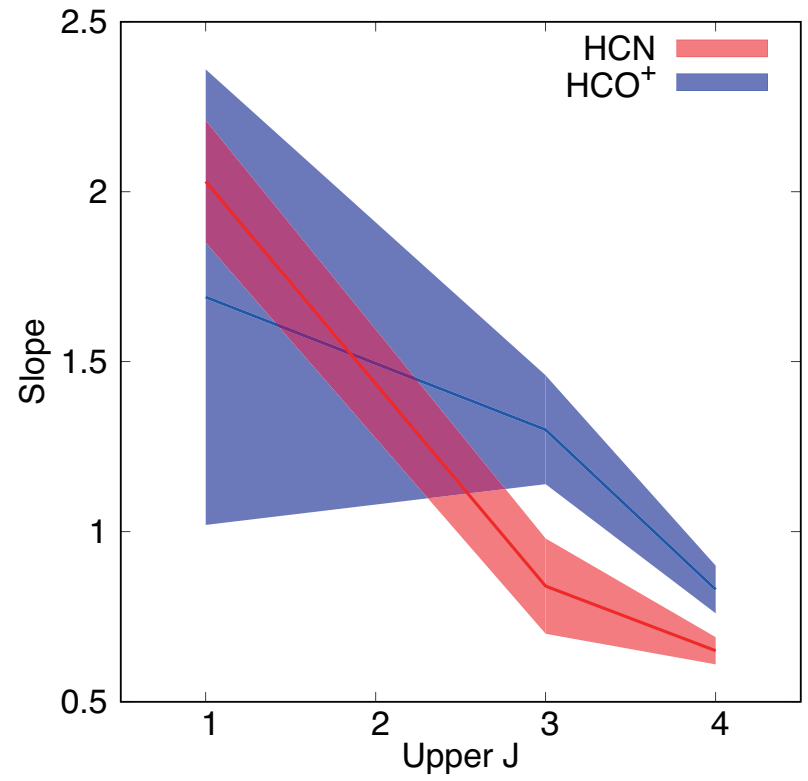

Figure 14. The index of the log-log plots shown in Figure 13. Red and blue tracks correspond to $\mathrm{HCN}$ and $\mathrm{HCO}^{+}$indices, respectively. $1 \sigma$ error ranges of the fitting are shown as colorized areas.

2006). In contrast to the SFEs, $f_{\text {dense }}$ peaks at Region 8 . The Overlap region has 1.5-2 times larger $f_{\text {dense }}$ than Region 1-3 and 11. The eastern nucleus is the site of young massive starburst, whereas the Overlap region is dominated by dense gas, and a site of moderate star formation, which has, for instance, $M_{\text {dense }}=(1.8 \pm 0.2) \times$ $10^{8} M_{\odot}$ (Region 6$)$ of fuel for future star formation.

\subsection{Turbulence-regulated Star Formation}

In order to investigate what physical mechanism governs the observable quantities of star formation at the filament of VV 114, we employ a turbulence-regulated star formation model (Krumholz \& McKee 2005; see also Usero et al. 2015 for an application to observing data). A simple formulation of this model is useful to parameterize the observed quantities (i.e., $\mathrm{SFE}_{\mathrm{H}_{2}}$ and $f_{\text {dense }}$ ) with molecular cloud properties. The equations are,

$$
\begin{aligned}
\mathrm{SFE}_{\mathrm{H}_{2}} & =\epsilon_{\mathrm{SF}} \frac{(\mathcal{M} / 100)^{-0.32}}{\tau_{\mathrm{ff}}(\bar{n})}, \\
f_{\text {dense }} & =\frac{1}{2}\left[1+\operatorname{erf}\left(\frac{\sigma_{\mathrm{PDF}}^{2}-2 \log \left(n_{\text {dense }} / \bar{n}\right)}{2^{3 / 2} \sigma_{\mathrm{PDF}}}\right)\right], \\
\sigma_{\mathrm{PDF}}^{2} & \approx \log \left(1+\frac{3 \mathcal{M}^{2}}{4}\right)
\end{aligned}
$$

where $\epsilon_{\mathrm{SF}}$ is the efficiency depending on the virial state of the clouds, $\mathcal{M}$ is the Mach number, $\tau_{\mathrm{ff}}(\bar{n})$ is the freefall timescale $(\sqrt{3 \pi /(32 \bar{n} G)})$ for a given average volume density $(\bar{n})$, and $\sigma_{\mathrm{PDF}}$ is the width of the lognormal probability distribution function (PDF) of gas density within a cloud. This model explains the star formation in a virialized molecular cloud using two parameters, $\mathcal{M}$ and $\bar{n}$ : (1) When turbulence in a cloud is high (i.e., high $\mathcal{M}$ ), it inhibits star formation leading to low $\mathrm{SFE}_{\mathrm{H}_{2}}$, although it broadens the density PDF (i.e., increasing the fraction of HCN-emitting dense gas $=$ high $f_{\text {dense }}$ ). (2) When the average gas density $(\bar{n})$ increases, it shortens $\tau_{\mathrm{ff}}(\bar{n})$ (i.e., $\mathrm{SFE}_{\mathrm{H}_{2}}$ increases), and naturally results in higher $f_{\text {dense }}$. Throughout this Paper, we assume $n_{\text {dense }}=2.8$ $\times 10^{5} \mathrm{~cm}^{-3}$ and $\epsilon_{\mathrm{SF}}=0.28 \%$, which are the same values as those used in Usero et al. (2015).

The observed $\log \mathrm{SFE}_{\mathrm{H}_{2}}$ against $\log f_{\text {dense }}$ is plotted in Figure 16. As already seen in Figure 15, data points at the Overlap region shows systematically high $f_{\text {dense }}$ and low $\mathrm{SFE}_{\mathrm{H}_{2}}$. We overlaid the turbulence-regulated star formation model ( $\mathcal{M}-\bar{n}$ grid) on this Figure. The grid clearly characterizes the Overlap region as a turbulent lower density $\operatorname{ISM}\left(\mathcal{M} \sim 5\right.$ and $\left.\bar{n} \sim 10^{4.0} \mathrm{~cm}^{-3}\right)$ relative to the eastern nucleus $\left(\mathcal{M} \sim 2\right.$ and $\left.\bar{n} \sim 10^{4.5} \mathrm{~cm}^{-3}\right)$. The absolute values are uncertain because of the assumed conversion factors to derive the surface densities and the assigned SFR tracer. For example, when we use $8.4 \mathrm{GHz}$ continuum emission as the SFR tracer (instead of the $110 \mathrm{GHz}$ continuum), $\mathrm{SFE}_{\mathrm{H}_{2}}$ decreases by a factor of $\sim 2$, hence $\mathcal{M}(\bar{n})$ tends to increase (decrease). The effect of uncertainties of the conversion factors on SFEs will be discussed later. However, since the general trend does not change regardless of the employed SFR tracer or adopted conversion factor, the relative differences between the eastern nucleus and the Overlap region also do not change.

Large-scale shocks driven by the interaction between the progenitor's galaxies where proposed by Saito et al. (2017a), which causes an enhancement of methanol abundance at the Overlap region. We now suggest that the starburst is induced by this shock. Molecular clouds at the Overlap region of VV 114 are compressed by violent merger-induced shocks, and then the shock-induced intracloud turbulence have started to dissipate, which in part will form new stars. This view is consistent with the scenario of widespread star formation at the early-to-mid stages of major mergers predicted by numerical simulations (e.g., Saitoh et al. 2009). The eastern nucleus already shows intense star formation activity, which might be due to efficient gas inflow towards the nucleus at the early-stage of a merger (Iono et al. 2004b).

Another model, the so-called density threshold model (e.g., Gao \& Solomon 2004a,b), suggests that the rate at which molecular gas turns into stars depends on the dense gas mass within a molecular cloud (i.e., $\Sigma_{\text {SFR }} \propto$ $\Sigma_{\text {dense }}=f_{\text {dense }} \Sigma_{\mathrm{H}_{2}}$; e.g., Lada et al. 2012), and thus it can simply explain the observed linear correlation between $L_{\mathrm{IR}}$ and $L_{\mathrm{HCN}(1-0)}^{\prime}$. It, in turn, predicts a linear correlation between $\mathrm{SFE}_{\mathrm{H}_{2}}\left(=\Sigma_{\mathrm{SFR}} / \Sigma_{\mathrm{H}_{2}}\right)$ and $f_{\text {dense }}$. However, as we can see in Figure 16, VV 114 data do not show such a positive linear correlation (the correlation coefficient is -0.55$)$, showing that the density threshold model is not appropriate for the VV 114 data. The density threshold model also predicts constant $\mathrm{SFE}_{\text {dense }}$ from Galactic star-forming region to extragalactic starforming region (i.e., $\Sigma_{\text {SFR }} \propto \Sigma_{\text {dense }}$ ), although the filament of VV 114 shows a decreasing trend towards the west (Figure 15).

We note that this result does not change by adopting different conversion factors to derive $\Sigma_{\text {dense }}$ and $\Sigma_{\mathrm{H}_{2}}$. Considering that $\alpha_{\mathrm{HCN}}$ scales similarly as $\alpha_{\mathrm{CO}}$, and $\alpha_{\mathrm{CO}}$ is a few times lower at the nuclear regions than in other quiescent regions of galaxies (Sandstrom et al. 2013), $\alpha_{\mathrm{HCN}}$ should not be larger at the eastern nucleus of 

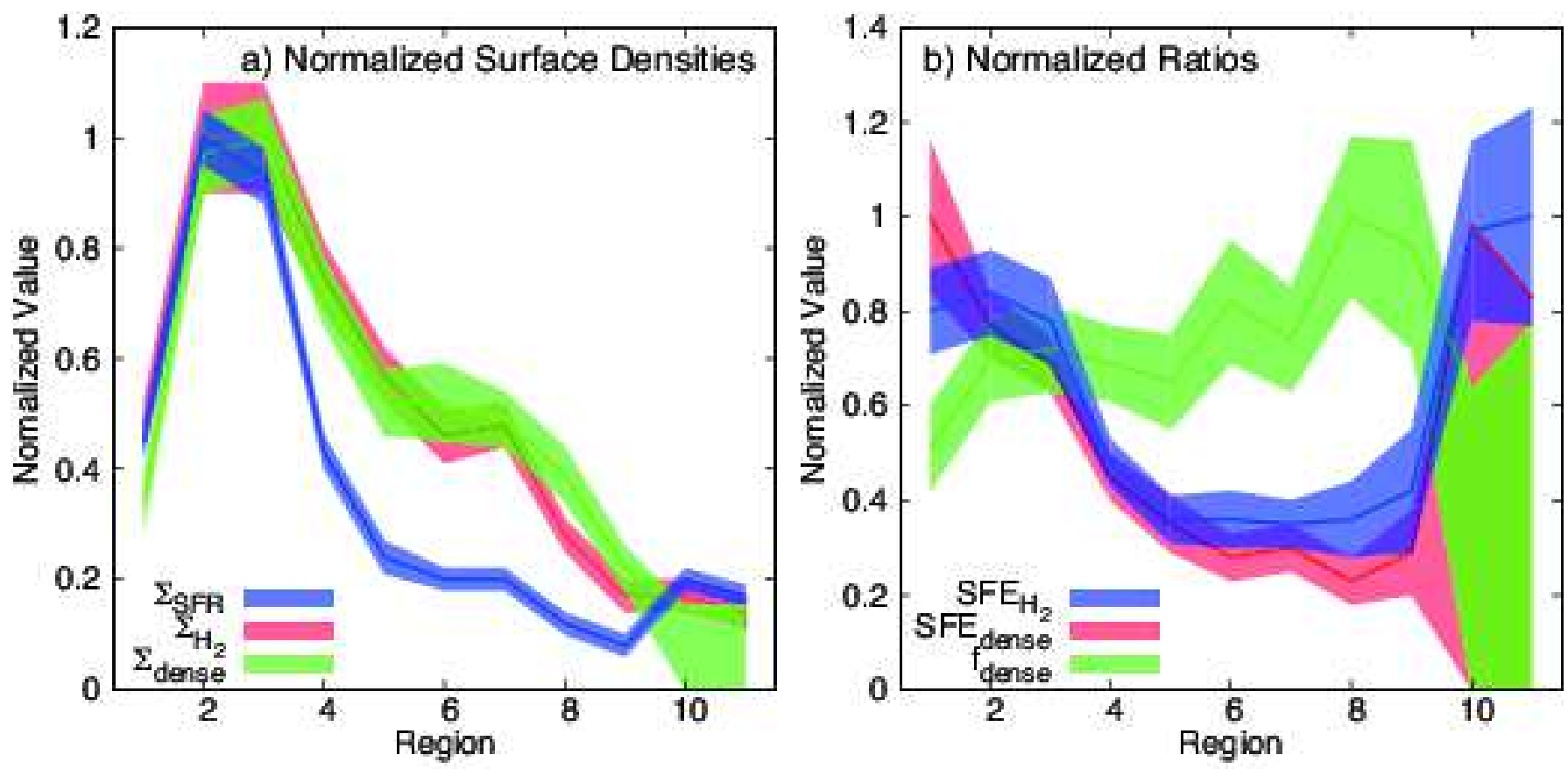

Figure 15. (a) Normalized distributions of $\Sigma_{\mathrm{SFR}}$ (blue), $\Sigma_{\mathrm{H}_{2}}$ (red), and $\Sigma_{\text {dense }}$ (green) along the filament of VV 114. The absolute values

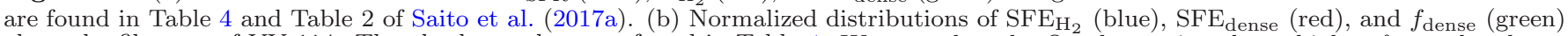
along the filament of VV 114. The absolute values are found in Table 4. We note that the Overlap region shows higher $f_{\text {dense }}$, but lower $\mathrm{SFE}_{\mathrm{H}_{2}}$ and $\mathrm{SFE}_{\text {dense }}$ (see text in detail).

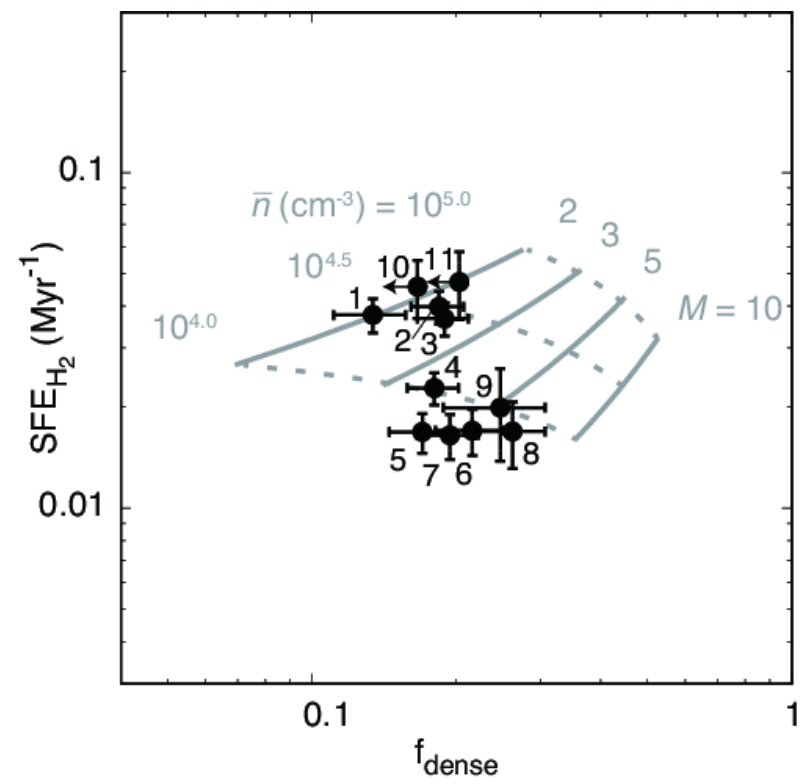

Figure 16. $\mathrm{SFE}_{\mathrm{H}_{2}}$ plots against $f_{\text {dense. }}$. Aperture IDs are shown for each data point. Grids of the turbulence-regulated star formation model are shown in grey curves (Krumholz \& McKee 2005; Usero et al. 2015). Model curves with constant Mach number $(\mathcal{M})$ or constant average density $(\bar{n})$ are shown in solid or dashed curves, respectively.

VV 114 than at the Overlap region. In order to get a constant $\mathrm{SFE}_{\text {dense }}$ along the filament of VV 114, we could need more (less) $\Sigma_{\text {dense }}$ at the eastern nucleus (Overlap region), resulting in 2-3 times larger $\alpha_{\mathrm{HCN}}$ at the eastern nucleus, which is not an applicable value.

Similar to $\mathrm{SFE}_{\text {dense, }}$, the difference of $\mathrm{SFE}_{\mathrm{H}_{2}}$ between the nucleus and the Overlap region tends to increase when adopting plausible conversion factors. We used the formulation described in Scoville et al. (2016) to derive $\Sigma_{\mathrm{H}_{2}}$ from $880 \mu \mathrm{m}$ continuum flux (see Saito et al. 2017a), assuming a constant $T_{\text {dust }}$ of $25 \mathrm{~K}$. However, the intense nuclear starburst at the eastern nucleus may result in warmer gas temperature than that for the Overlap region (see Section 5.5). Since the derived $\Sigma_{\mathrm{H}_{2}}$ decreases with increasing $T_{\text {dust }}, \mathrm{SFE}_{\mathrm{H}_{2}}$ tends to increase (decrease) at the eastern nucleus (Overlap region), which enlarges the observed $\mathrm{SFE}_{\mathrm{H}_{2}}$ gradient along the filament. In summary, the systematic uncertainties of the conversion factors do not change the trend seen in Figure 16.

\subsection{Line Ratios vs. $\Sigma_{\mathrm{SFR}}$}

Here we examine the dependence of dense gas excitation ratios on $\Sigma_{\mathrm{SFR}}$ using the measured data along the filament of VV 114 (see Table 2), as previously done for CO excitation (Tsai et al. 2012; Rosenberg et al. 2015; Kamenetzky et al. 2016). As shown in Figure 17, all excitation ratios measured in VV 114 correlate well with $\Sigma_{\mathrm{SFR}}$, although Region 1 (i.e., AGN position) clearly deviates from the correlation. The correlation coefficients are $0.66,0.70,0.67$, and 0.70 , for $\mathrm{HCN}(3-2) / \mathrm{HCN}(1-$ $0)$ (Figure 17a), HCN (4-3)/ $\mathrm{HCN}(1-0)$ (Figure 17b), $\mathrm{HCO}^{+}(3-2) / \mathrm{HCO}^{+}(1-0)$ (Figure $\left.17 \mathrm{c}\right)$, and $\mathrm{HCO}^{+}(4-$ $3) / \mathrm{HCO}^{+}(1-0)$ (Figure 17d), respectively, although the coefficients become $0.97,0.99,0.96$, and 0.95 , respectively when excluding Region 1 . This indicates that the excitation condition at Region 1 is clearly different from that at other regions in the filament, and star formation activity may govern dense gas excitation except for Region 1. A conceivable explanation of the unusual excitation found in Region 1 is the influence of an AGN. Region 1 shows the highest excitation ratios in VV 114 but modest $\Sigma_{\mathrm{SFR}}$. The $\Sigma_{\mathrm{SFR}}$ at Region 1, derived by 

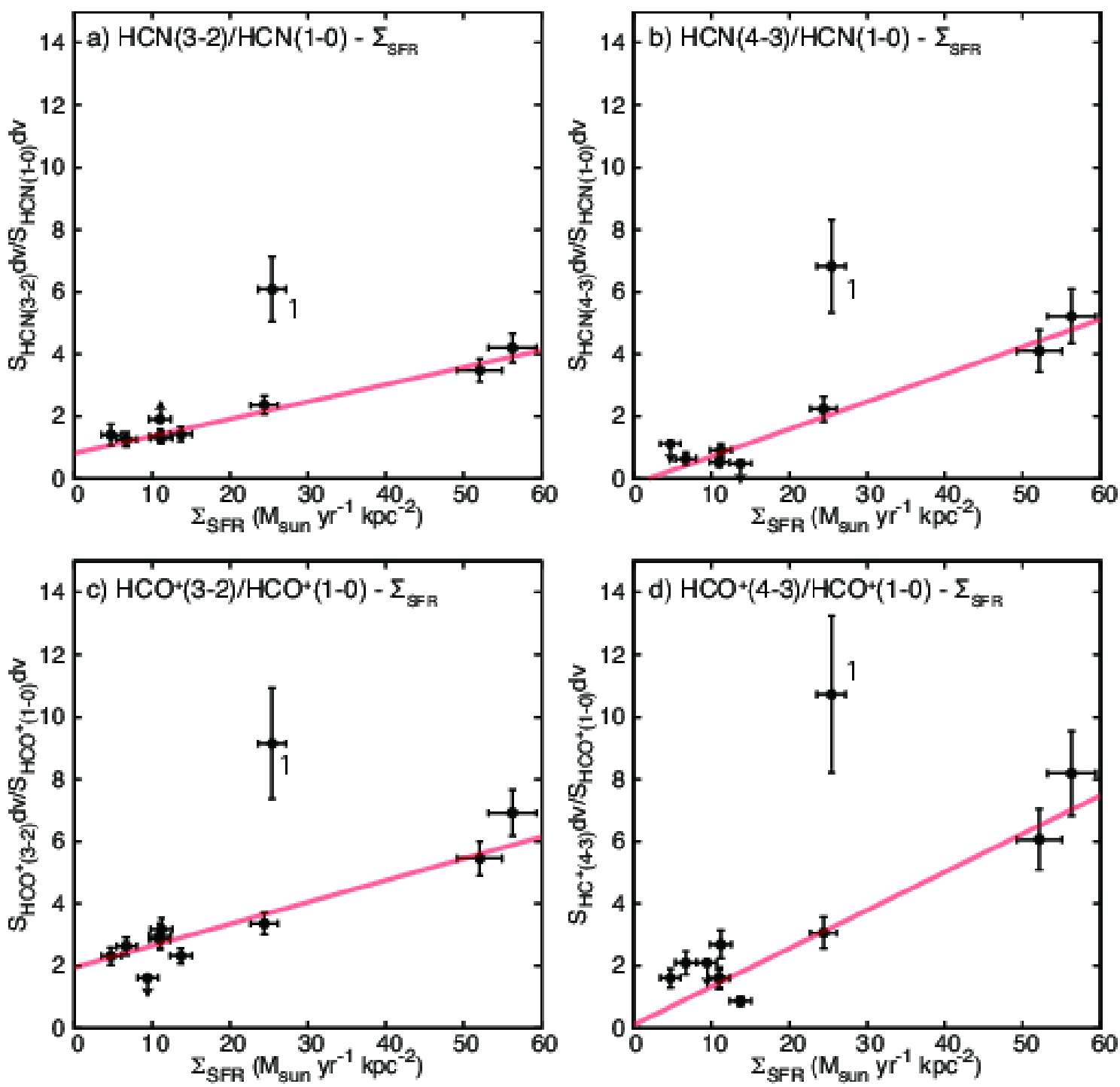

Figure 17. Excitation ratio plots against $\Sigma_{\mathrm{SFR}}$ : (a) $\mathrm{HCN}(3-2) / \mathrm{HCN}(1-0)$ ratio, (b) $\mathrm{HCN}(4-3) / \mathrm{HCN}^{(1-0)} \mathrm{ratio}$ (c) $\mathrm{HCO}^{+}(3-$ $2) / \mathrm{HCO}^{+}(1-0)$ ratio, and $(\mathrm{d}) \mathrm{HCO}^{+}(4-3) / \mathrm{HCO}^{+}(1-0)$ ratio. Data point at the AGN position is denoted as 1 . The best fitted lines except for Region 1 are shown as a red line.

using $110 \mathrm{GHz}$ continuum emission, may be an upper limit because of the non-thermal contribution from the putative AGN (see Saito et al. 2017a), so Region 1 deviates more and more when excluding the possible AGN contribution. This is consistent with the results from high-resolution observations of multiple molecular lines toward the nearest type 2 AGN host LIRG NGC 1068 (García-Burillo et al. 2014; Viti et al. 2014). The circumnuclear disk of NGC 1068, whose ISM is thought to be dominantly affected by the central AGN, has at least a few times higher excitation ratios of $\mathrm{CO}, \mathrm{HCN}$, and $\mathrm{HCO}^{+}$than in the starburst ring with hundred pc scale.

The $\mathrm{HCN} / \mathrm{HCO}^{+}$line ratios show a similar trend against $\Sigma_{\mathrm{SFR}}$. The correlation coefficients are $0.46,0.57$, and 0.62 , for $\mathrm{HCN}(1-0) / \mathrm{HCO}^{+}(1-0)$ (Figure 18a), $\mathrm{HCN}(3-2) / \mathrm{HCO}^{+}(3-2)$ (Figure $\left.18 \mathrm{~b}\right)$, and $\mathrm{HCN}(4-$ $3) / \mathrm{HCO}^{+}(4-3)$ (Figure $18 \mathrm{c}$ ), respectively, although the coefficients become $0.85,0.87$, and 0.93 , respectively when excluding Region 1 . Since, for a given $J$, HCN has a few times higher critical density than $\mathrm{HCO}^{+}$(Shirley
2015), the strong correlation between $\mathrm{HCN} / \mathrm{HCO}^{+}$ratios and $\Sigma_{\text {SFR }}$ is naturally explained by FUV heating from star-forming regions as with the correlation between excitation ratios and $\Sigma_{\text {SFR }}$. On the other hand, Region 1 clearly deviates from trends found in all line ratio plots against $\Sigma_{\mathrm{SFR}}$, suggesting the presence of another mechanisms to boost the observed line ratios (i.e., AGN).

We note that Figures 17 and 18 can be also regarded as luminosity ratio plots against FIR luminosity because all data points have similar distance, aperture size, and filling factor.

\subsection{Radiative Transfer Modeling under LTE}

Here we derive excitation temperature $\left(T_{\text {rot }}\right)$ and column density $\left(N_{\text {tot }}\right)$ at each aperture position in VV 114 using a single component rotation diagram method, assuming optically-thin gas and LTE conditions. Recent multiple CO transitions have revealed that nearby IRbright galaxies (e.g., Downes \& Solomon 1998; Zhu et al. 2003; Iono et al. 2007; Zhu et al. 2007; Sliwa et al. 2012; 

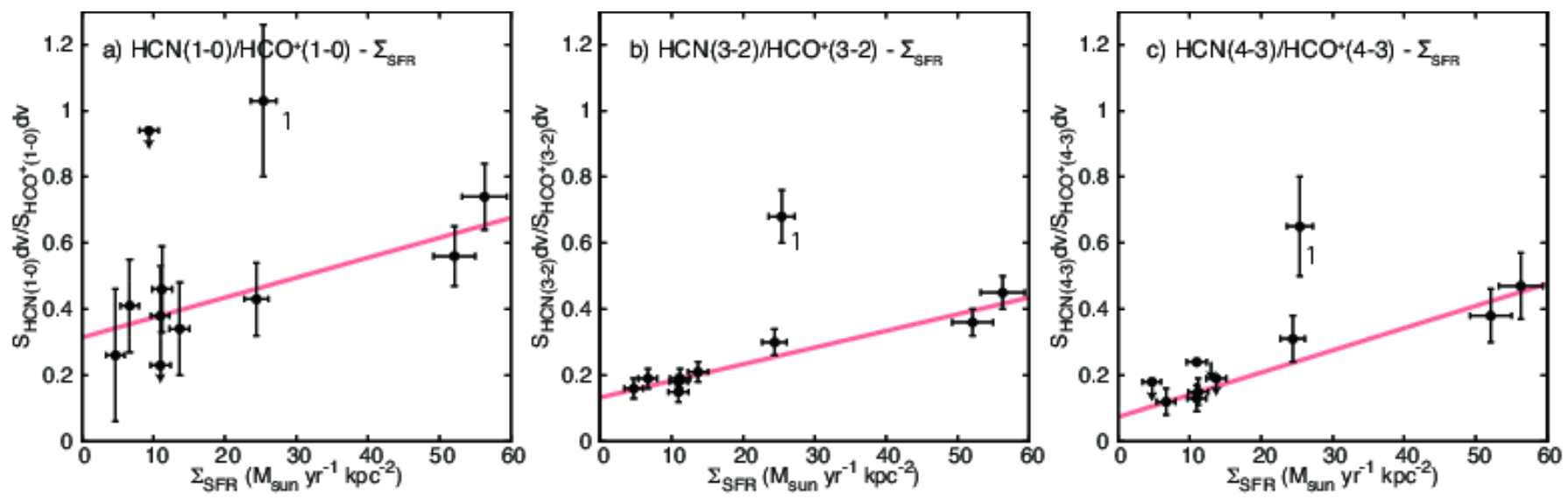

Figure 18. $\mathrm{HCN} / \mathrm{HCO}^{+}$ratio plots against $\Sigma_{\mathrm{SFR}}$ : (a) $\mathrm{HCN}(1-0) / \mathrm{HCO}^{+}(1-0)$ ratio, (b) $\mathrm{HCN}(3-2) / \mathrm{HCO}^{+}(3-2)$ ratio, and (c) $\mathrm{HCN}(4-3) / \mathrm{HCO}^{+}(4-3)$ ratio. Data point at the AGN position is denoted as 1 . The best fitted lines except for Region 1 are shown as a red line.

Saito et al. 2015, 2017b) show CO (1-0) emission with moderate optical depth $(\sim 1)$. Considering 1-2 orders of magnitude weaker $\mathrm{HCN}(1-0)$ and $\mathrm{HCO}^{+}(1-0)$ emission relative to $\mathrm{CO}(1-0)$ in such IR-bright galaxies (Gao \& Solomon 2004a,b; Privon et al. 2015), it is reasonable to assume optically-thin $\mathrm{HCN}(1-0)$ and $\mathrm{HCO}^{+}(1-0)$ lines in VV 114. In Section 5.5.2, we examine the appropriateness of the optically-thin and the single component assumptions.

\subsubsection{Rotation Diagram Analysis}

We fitted the observed flux densities by using the following equation (e.g., Goldsmith \& Langer 1999; Watanabe et al. 2014; Mangum \& Shirley 2015),

$$
\begin{aligned}
W_{\nu}= & \frac{8 \pi^{3} S \mu_{0}^{2} \nu N_{\text {tot }}}{3 k Q_{\text {rot }}}\left\{1-\frac{\exp \left(h \nu / k T_{\text {rot }}\right)-1}{\exp \left(h \nu / k T_{\mathrm{bg}}\right)-1}\right\} \\
& \times \exp \left(-\frac{E_{\mathrm{u}}}{k T_{\text {rot }}}\right)
\end{aligned}
$$

where $W_{\nu}$ is the integrated intensity in units of $\mathrm{K} \mathrm{km} \mathrm{s}^{-1}$ (proportional to the upper state column density, $N_{\mathrm{u}}$ ), $S$ is the line strength, $\mu_{0}$ is the dipole moment, $\nu$ is the frequency of the transition, $Q_{\text {rot }}$ is the rotational partition function, $k$ is the Boltzmann constant, $h$ is the Planck constant, $T_{\mathrm{bg}}$ is the cosmic microwave background temperature $(=2.73 \mathrm{~K})$, and $E_{\mathrm{u}}$ is the upper state energy. The molecular line database Splatalogue ${ }^{17}$ and the Cologne Database for Molecular Spectroscopy $\left(\mathrm{CDMS}^{18}\right.$; Müller et al. 2001, 2005) were used to get the transition parameters necessary for the calculation. For regions without multiple $J$ detections, we used an averaged $T_{\text {rot }}$ between Region 3 and 9 , where are not contaminated by the putative AGN.

Figure 19a shows the fitting result for Region 1 as an example. The data appear to be well fitted by a single component, although all regions show that $J=3$ 2 ( $J=1-0$ and $4-3)$ flux is overestimated (underestimated). Some possibilities to explain this trend will be discussed in Section 5.5.2. The derived $T_{\text {rot }}$ and $N_{\text {tot }}$ for $\mathrm{HCN}\left(T_{\mathrm{HCN}}\right.$ and $\left.N_{\mathrm{HCN}}\right)$ and $\mathrm{HCO}^{+}\left(T_{\mathrm{HCO}}+\right.$ and $\left.N_{\mathrm{HCO}^{+}}\right)$

\footnotetext{
17 http://www.splatalogue.net/

18 http://www.astro.uni-koeln.de/cdms/catalog\#partition
}

are listed in Table 5 and shown in Figures $19 \mathrm{~b}$ and $19 \mathrm{c}$. Both $T_{\mathrm{HCN}}$ and $T_{\mathrm{HCO}^{+}}$peak at Region $1(10.2 \pm 2.5 \mathrm{~K}$ and $12.3 \pm 2.8 \mathrm{~K}$, respectively) and decrease towards the western side of the filament. The derived temperature of 5-8 $\mathrm{K}$ at the Overlap region is consistent with the $T_{\text {rot }}$ derived from rotational transitions of methanol $\left(T_{\mathrm{CH}_{3} \mathrm{OH}}=6-9 \mathrm{~K}\right.$; Saito et al. 2017a). We compare the derived $T_{\mathrm{HCN}}$ and $T_{\mathrm{HCO}^{+}}$with $\Sigma_{\mathrm{SFR}}$ in Figure 20 . This figure is more straightforward to interpret than the excitation ratio plots (Figure 17) to understand the energetics of dense gas, because some effects controlling the observed line ratios (e.g., optical depth, column density, and fractional abundance) are decomposed. Both $T_{\mathrm{HCN}}$ and $T_{\mathrm{HCO}}+$ correlate with $\Sigma_{\mathrm{SFR}}$, except for Region 1. The correlation coefficients are 0.71 and 0.63 for $T_{\mathrm{HCN}}$ and $T_{\mathrm{HCO}}{ }^{+}$, respectively, whereas, without Region 1 data, those are 0.96 and 0.94 , respectively. Those correlations clearly show that $\mathrm{HCN}$ and $\mathrm{HCO}^{+}$molecules are excited by star-forming activities in the filament of VV 114, and Region 1 needs additional efficient heating mechanisms. This explanation is consistent with $\mathrm{CO}$ excitation in (U)LIRGs (Greve et al. 2014; Kamenetzky et al. 2016), indicating that, in general, both diffuse and dense molecular ISM have a similar excitation condition (but see, e.g., Sakamoto et al. 2013; Aalto et al. 2015b; Imanishi et al. 2016b, which show the importance of radiative (IR) pumping for higher- $J \mathrm{HCN}$ and $\mathrm{HNC}$ ).

The derived dense gas column densities (Figure 19c) peak at Region 3 and slightly decrease towards the west, that is, the eastern nucleus contains warmer and more massive dense gas than the Overlap region. However, when we see the fractional abundances relative to $\mathrm{H}_{2}$ (e.g., $X_{\mathrm{HCN}}=N_{\mathrm{HCN}} / N_{\mathrm{H}_{2}}$ ), the trend changes. $X_{\mathrm{HCN}}$ shows a remarkably flat distribution along the filament, whereas $X_{\mathrm{HCO}}+$ shows a slightly increasing trend towards the west. These are the first measurements of spatiallyresolved $X_{\mathrm{HCN}}$ and $X_{\mathrm{HCO}^{+}}$for a LIRG. The average $X_{\mathrm{HCN}}$ and $X_{\mathrm{HCO}}+$ are $(3.5 \pm 0.7) \times 10^{-9}$ and $(8.9 \pm 1.4)$ $\times 10^{-9}$, respectively. Those fractional abundances are similar to other measurements for Galactic star-forming regions and nearby bright galaxies (e.g., Blake et al. 1987; Krips et al. 2008). Next, we divide $N_{\mathrm{HCN}}$ by $N_{\mathrm{HCO}}+$ to see the spatial distribution of the $\mathrm{HCN} / \mathrm{HCO}^{+}$abundance ratio, $X_{\mathrm{HCN}} / X_{\mathrm{HCO}^{+}}$, as shown in Figure 21. Al- 

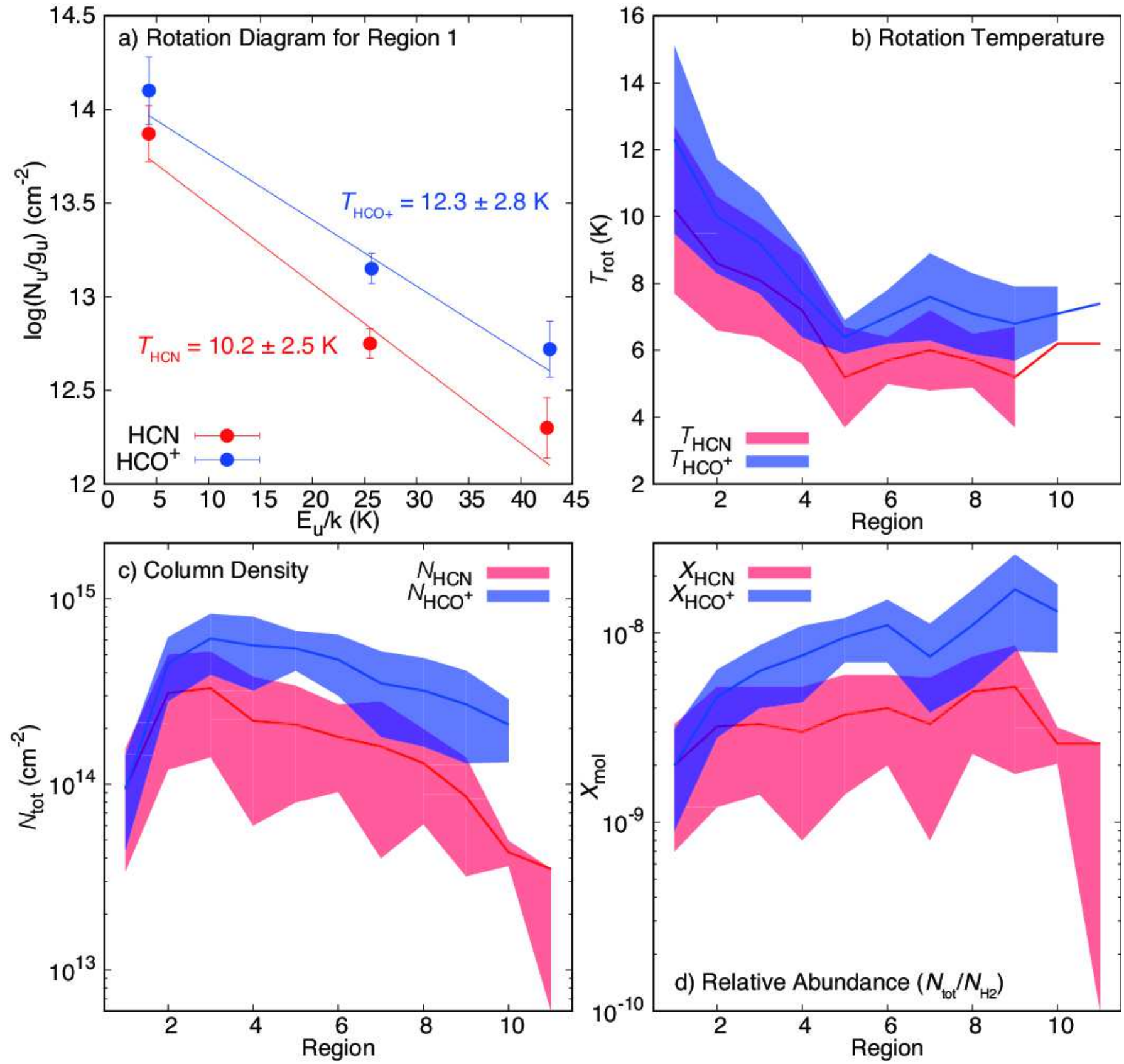

Figure 19. (a) Rotation diagrams of $\mathrm{HCN}$ and $\mathrm{HCO}^{+}$for Region 1 . The lines show the best fitted linear functions. The derived rotation temperature $\left(T_{\text {rot }}\right)$ values are also shown. (b) $T_{\text {rot }}$ obtained for each aperture along the filament of VV 114 . $1 \sigma$ error ranges of the fitting are shown as shaded areas. Values without error indicate the nominal $T_{\text {rot. }}$ (c) Column density $\left(N_{\text {tot }}\right)$ along the filament. (d) Fractional abundance relative to $\mathrm{H}_{2}\left(X_{\mathrm{mol}}\right)$ along the filament.

though the errors are large, the abundance ratio tends to peak at Region 1. Also, the $\mathrm{HCN}(1-0) / \mathrm{HCO}^{+}(1-0)$ flux ratio shows a similar trend (Figure 12) from 1 (Region 1 ) to 0.2 (Region 10), indicating that the opticallythin approximation for $\mathrm{HCN}(1-0)$ and $\mathrm{HCO}^{+}(1-0)$ lines is reasonable assumption in this case.

The same analysis for the AGN peak position (E0) shows that $T_{\mathrm{HCN}}=9.8 \pm 1.6 \mathrm{~K}, N_{\mathrm{HCN}}=(8.0 \pm 3.8)$ $\times 10^{13} \mathrm{~cm}^{-2}, T_{\mathrm{HCO}^{+}}=10.9 \pm 2.4 \mathrm{~K}, N_{\mathrm{HCO}^{+}}=(5.4 \pm$ $3.3) \times 10^{13} \mathrm{~cm}^{-2}$, and $X_{\mathrm{HCN}} / X_{\mathrm{HCO}^{+}}=1.5 \pm 1.2(\mathrm{Ta}-$ ble 5). The putative AGN position of VV 114 shows a relatively high $X_{\mathrm{HCN}} / X_{\mathrm{HCO}^{+}}$, resulting in observed high
$\mathrm{HCN}(1-0) / \mathrm{HCO}^{+}(1-0)$ flux ratio. Higher $\mathrm{HCN} / \mathrm{HCO}^{+}$ ratios in higher- $J$ seen at the AGN position is due to the combination effect of the high $X_{\mathrm{HCN}} / X_{\mathrm{HCO}^{+}}$and comparably high rotation temperatures between both molecules $\left(T_{\mathrm{HCN}} \simeq T_{\mathrm{HCO}^{+}}\right)$. Similar rotation temperatures keep the ratio between the upper state column densities of $\mathrm{HCN}$ and $\mathrm{HCO}^{+}$for a given $J$, so the observed $\mathrm{HCN} / \mathrm{HCO}^{+}$ratio SLED seems to be flat close to the AGN position (Figure 12). Other regions along the filament show relatively low $X_{\mathrm{HCN}} / X_{\mathrm{HCO}^{+}}(\lesssim 0.7)$ and low rotation temperatures $(\lesssim 10 \mathrm{~K})$. Lower $\mathrm{HCN}$ temperatures $\left(T_{\mathrm{HCN}}<T_{\mathrm{HCO}^{+}}\right)$result in the steeper shape 


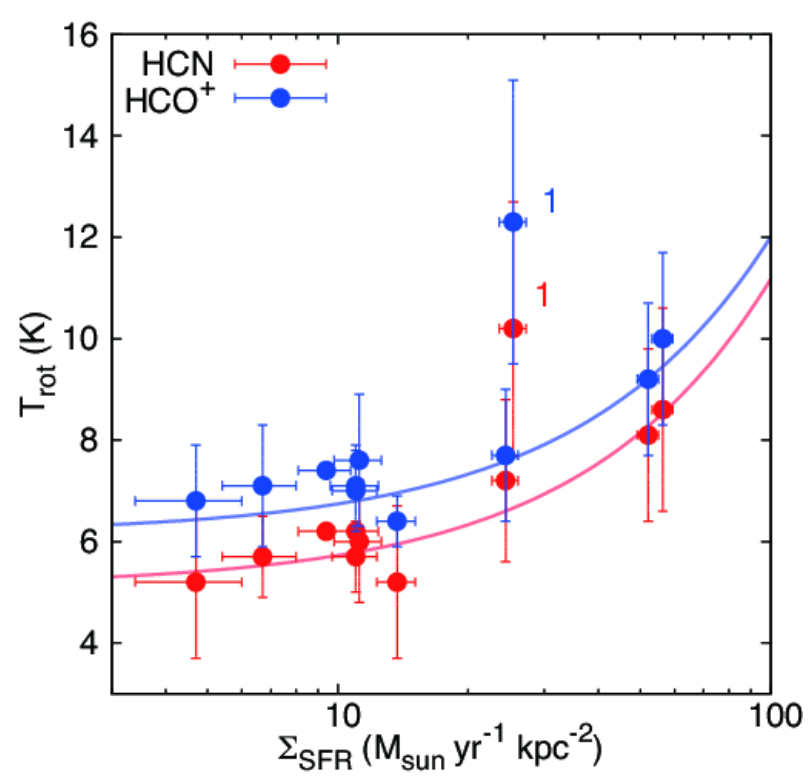

Figure 20. $T_{\text {rot }}$ plot against $\Sigma_{\mathrm{SFR}}$. Data point at the AGN position is denoted as 1 . The lines show the best fitted linear functions (excluding Region 1).

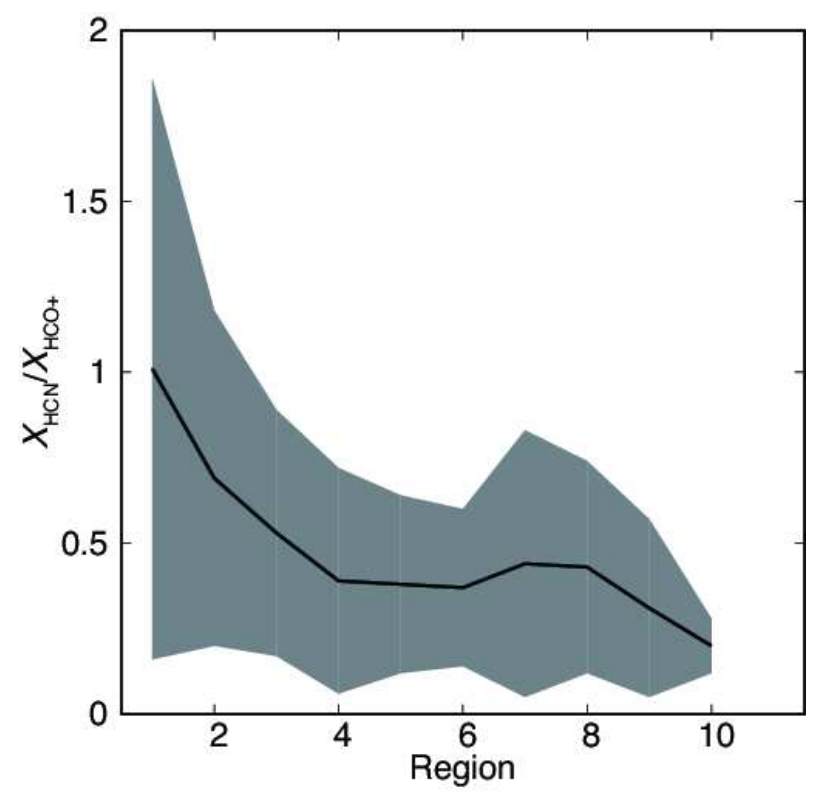

Figure 21. $X_{\mathrm{HCN}} / X_{\mathrm{HCO}}+$ along the filament of VV 114. The black line shows the best fit value. The shaded area shows the $1 \sigma$ error range.

of the $\mathrm{HCN} / \mathrm{HCO}^{+}$ratio SLED.

\subsubsection{Two-component ISM?}

Here, we discuss the effect of optical depth on the rotation diagram analysis. According to the LTE radiative transfer calculation described by Goldsmith \& Langer (1999), the transition of maximum opacity for a given linear molecule can be written as,

$$
J_{\max \tau}=4.6 \sqrt{T / B_{0}}
$$

where $T$ is the cloud temperature in Kelvin and $B_{0}$ is the rotation constant in $\mathrm{GHz}$. For $\mathrm{HCN}$ molecule $\left(B_{0}=\right.$
44.31597 GHz) with $T=10-20 \mathrm{~K}, J_{\max \tau}=2-3$. For the sake of simplicity, we ignore the background radiation term. In the limit $h B_{0} / k T<1$ (i.e., $0.048 B_{0}<T$ ), the ratio of the optical depth of the most optically thick transition $\left(\tau_{\max }\right)$ to that of the $J=1-0$ transition $\left(\tau_{1,0}\right)$ can be expressed as,

$$
\frac{\tau_{\max }}{\tau_{1,0}}=\frac{T}{0.048 e B_{0}} .
$$

This indicates that, for HCN molecule with $T=10-20 \mathrm{~K}$, optical depth at $J_{\text {upp }} \sim 3$ is 1.7-3.5 times larger than that at $J_{\text {upp }}=1$.

On the rotation diagram (for example, Figure 19a), data points should be aligned with a single straight line when the observed transitions are optically-thin under LTE. The expected deviation of the apparent upper state column density at $J_{\max \tau}$ from the optically-thin straight line can be estimated using the equation below.

$$
N_{\mathrm{u}}=\frac{\tau_{\max }}{1-e^{-\tau_{\max }}} N_{\mathrm{u}}^{\mathrm{thin}}
$$

where $N_{\mathrm{u}}^{\text {thin }}$ is the apparent upper state column density attenuated by line opacity $\left(\tau_{\max }\right)$ and $N_{\mathrm{u}}$ is the opacitycorrected upper state column density. When $\tau \rightarrow 0$ (i.e., optically-thin limit), $N_{\mathrm{u}}^{\text {thin }}$ becomes $N_{\mathrm{u}}$. The apparent deviation at $J=3-2$ from the straight line through $J=$ $1-0$ and $4-3$ is $N_{\mathrm{u}} / N_{\mathrm{u}}^{\text {thin }} \sim 10^{13.0} / 10^{12.75} \sim 1.78$ (Figure $19 \mathrm{a}$ ), which corresponds to $\tau_{\max } \sim 1.3$. This $\tau_{\max }$ is a lower limit because here we assumed optically-thin $J=$ 1-0 and 4-3 emissions to derive $N_{\mathrm{u}}=10^{13.0} \mathrm{~cm}^{-2}$. Using $\tau_{\max } / \tau_{1,0}=1.7-3.5, \tau_{1,0}$ is $\gtrsim 0.4-0.8$. Although this is a rough estimate, it is consistent with $\tau_{1,0}=0.3-1.4$ derived by the non-LTE modeling for $\mathrm{HCN}$ and $\mathrm{HCO}^{+}$ transitions (Krips et al. 2008), indicating that the moderate optical depth at $J=3-2\left(\tau_{\max } \gtrsim 1.3\right)$ may drive the apparent two-component excitation seen in the rotation diagram. This discussion can also be applied to $\mathrm{HCO}^{+}$, which has similar excitation parameters to $\mathrm{HCN}$. We conclude that the apparent two-component excitation condition seen in the rotation diagrams of $\mathrm{HCN}$ and $\mathrm{HCO}^{+}$can be explained by finite optical depths, which peak at $J_{\text {upp }} \sim 3$. For much higher- $J$ HCN $\left(J_{\text {upp }} \sim 15\right)$, radiative pumping rather than collision becomes more important to explain the level population, resulting in a true two-component ISM (e.g., Rangwala et al. 2011). Observations of other rotational transitions are essential to investigate the appropriateness for other assumptions (e.g., non-LTE excitation).

\section{CONCLUSION}

We present new high-resolution $\left(0 ., 2-1^{\prime \prime} .5\right)$ images of multiple $\mathrm{HCN}$ and $\mathrm{HCO}^{+}$transitions $(J=1-0,3-2$, and $4-3)$, HNC $J=1-0$, CS $J=7-6$, and continuum emissions toward the nearby luminous infrared galaxy VV 114 with ALMA bands 3, 6, and 7. We map the spatial distribution of the three $\mathrm{HCN}$ and $\mathrm{HCO}^{+}$transitions for a LIRG for the first time. We summarize the main results of this Paper as follows:

1. All line and continuum images, except for $\mathrm{HCN}(4-$ 3 ) and CS (7-6) lines (probably because of their high critical densities and low flux densities), show an extended clumpy filament ( $\sim 6 \mathrm{kpc}$ length) 
across the progenitor's galaxy nuclei of VV 114, which has been previously mapped through many molecular and ionized gas tracers. With our highresolution Band 7 continuum images $(\sim 80$ pc resolution), we found that the filament has a narrow width $(\lesssim 200 \mathrm{pc})$. Such filamentary structure is predicted by numerical simulations of major mergers.

2. Both excitation ratios (i.e., $\mathrm{HCN}\left(J_{\mathrm{u}}-J_{1}\right) / \mathrm{HCN}(1-$ $0)$ ) and $\mathrm{HCN} / \mathrm{HCO}^{+}$ratios (e.g., $\mathrm{HCN}\left(J_{\mathrm{u}}-\right.$ $\left.\left.J_{1}\right) / \mathrm{HCO}^{+}\left(J_{\mathrm{u}}-J_{1}\right)\right)$ show higher values at the eastern nucleus, relative to the Overlap region between the nuclei of the two galaxies. We plot spatiallyresolved $\mathrm{HCN}$ and $\mathrm{HCO}^{+}$SLEDs for the first time, which clearly shows that the peaks are located at $J_{\text {upp }} \lesssim 3$ for the Overlap region whereas $J_{\text {upp }} \gtrsim 3$ for the eastern nucleus. The values in the $\mathrm{HCO}^{+}$ SLED are generally larger than those in the HCN SLED. The $\mathrm{HCN} / \mathrm{HCO}^{+}$ratio SLED shows a remarkably high, flat shape at the AGN position, while a decreasing trend with increasing $J_{\text {upp }}$ is seen in other regions.

3. Integrated fluxes $-\Sigma_{\text {SFR }}$ and flux ratios $-\Sigma_{\text {SFR }}$ plots of different $3^{\prime \prime}$ apertures along the filament show positive trends when excluding the putative AGN, indicating that star formation activity is closely related to surface density and excitation of dense gas. For both $\mathrm{HCN}$ and $\mathrm{HCO}^{+}$, we found that the slope of the $\log L_{\text {dense }}^{\prime}-\log L_{\text {IR }}$ plots decreases as $J$ increases. This can be explained by subthermalized gas excitation at the lower $\Sigma_{\mathrm{SFR}}$ $\left(L_{\mathrm{IR}}\right)$ regime and/or the presence of powerful heating source(s) at the higher $\Sigma_{\mathrm{SFR}}$ regime.

4. Furthermore, we estimate the surface densities of total $\mathrm{H}_{2}$ mass and dense gas mass, and then $\mathrm{SFE}_{\mathrm{H}_{2}}$ $\left(=\Sigma_{\mathrm{SFR}} / \Sigma_{\mathrm{H}_{2}}\right), \mathrm{SFE}_{\text {dense }}\left(=\Sigma_{\mathrm{SFR}} / \Sigma_{\text {dense }}\right)$, and $f_{\text {dense }}\left(=\Sigma_{\text {dense }} / \Sigma_{\mathrm{H}_{2}}\right)$, assuming constant conversion factors. Although both SFEs peak at the eastern nucleus and are lower at the Overlap region, $f_{\text {dense }}$ shows a different trend and peaks at the Overlap region. This trend becomes clearer when we adopt appropriate conversion factors. A simple so-called density threshold star formation model cannot reproduce these trends.

5. We examine a turbulence-regulated star formation model, and find that the Overlap region has more diffuse and turbulent dense gas properties relative to that of the eastern nucleus. Combined with the fact that methanol abundance in gas-phase at the Overlap region is elevated due to large-scale shocks, we suggest that collision between the progenitors of VV 114 has produced the filamentary structure and violent gas inflow toward the eastern nucleus is triggering both starburst and AGN activities. Merger-induced shocks, which can enhance the intracloud turbulence, dominates at the Overlap region, but dissipates to form new massive star-forming regions.

6. Radiative transfer analysis under LTE and optically-thin assumptions revealed that the rotation temperatures and column densities are higher at the eastern nucleus. Except for the AGN position, the rotation temperatures are clearly correlated to $\Sigma_{\mathrm{SFR}}$, indicating that dense gas excitation in the filament is dominantly governed by star formation activity. The fractional abundance of $\mathrm{HCN}$ relative to $\mathrm{H}_{2}$ is remarkably flat along the filament, showing that the HCN abundance might be insensitive to environmental effects in VV 114 (i.e., AGN, starbursts, and shocked Overlap region). The average fractional abundances of $\mathrm{HCN}$ and $\mathrm{HCO}^{+}$are $(3.5 \pm 0.7) \times 10^{-9}$ and $(8.9 \pm 1.4) \times 10^{-9}$, respectively.

7. The observed high $\mathrm{HCN} / \mathrm{HCO}^{+}$flux ratio at the eastern nucleus is likely due to a high $\mathrm{HCN} / \mathrm{HCO}^{+}$ abundance ratio, and the high excitation ratio there is potentially due to efficient heating by AGN activity.

The authors thank an anonymous referee for cements that improved the contents of this paper. TS and other authors thank ALMA staff for their kind support. TS acknowledges funding from the European Research Council (ERC) under the European Unions Horizon 2020 research and innovation programme (grant agreement No. 694343). This paper makes use of the following ALMA data: ADS/JAO.ALMA\#2011.0.00467.S, ADS/JAO.ALMA\#2013.1.00740.S,

ADS/JAO.ALMA\#2013.1.01057.S, and ADS/JAO.ALMA\#2015.1.00973.S. ALMA is a partnership of ESO (representing its member states), NSF (USA) and NINS (Japan), together with NRC (Canada), NSC and ASIAA (Taiwan), and KASI (Republic of Korea), in cooperation with the Republic of Chile. The Joint ALMA Observatory is operated by ESO, AUI/NRAO and NAOJ. This research has made use of the NASA/IPAC Extragalactic Database (NED) which is operated by the Jet Propulsion Laboratory, California Institute of Technology, under contract with the National Aeronautics and Space Administration.

\section{REFERENCES}

Aalto, S., Johansson, L. E. B., Booth, R. S., \& Black, J. H. 1991, A\&A, 249, 323

Aalto, S., Polatidis, A. G., Hüttemeister, S., \& Curran, S. J. 2002, A\&A, 381, 783

Aalto, S., Spaans, M., Wiedner, M. C., \& Hüttemeister, S. 2007, A\&A, 464, 193

Aalto, S. 2013, Molecular Gas, Dust, and Star Formation in Galaxies, 292, 199

Aalto, S., Garcia-Burillo, S., Muller, S., et al. 2015, A\&A, 574, A85

Aalto, S., Martín, S., Costagliola, F., et al. 2015, A\&A, 584, A42

Aladro, R., Martín, S., Riquelme, D., et al. 2015, A\&A, 579, A101

Armus, L., Mazzarella, J. M., Evans, A. S., et al. 2009, PASP, 121,559

Blake, G. A., Sutton, E. C., Masson, C. R., \& Phillips, T. G. 1987, ApJ, 315, 621

Condon, J. J. 1992, ARA\&A, 30, 575

Costagliola, F., Aalto, S., Rodriguez, M. I., et al. 2011, A\&A, 528, A30

Costagliola, F., Aalto, S., Sakamoto, K., et al. 2013, A\&A, 556, A66

Downes, D., \& Solomon, P. M. 1998, ApJ, 507, 615

Espada, D., Matsushita, S., Miura, R. E., et al. 2017, ApJ, 843, 136 
Gao, Y., \& Solomon, P. M. 2004a, ApJ, 606, 271

Gao, Y., \& Solomon, P. M. 2004b, ApJS, 152, 63

García-Burillo, S., Usero, A., Alonso-Herrero, A., et al. 2012 , A\&A, 539, A8

García-Burillo, S., Combes, F., Usero, A., et al. 2014, A\&A, 567, A125

Goldsmith, P. F., \& Langer, W. D. 1999, ApJ, 517, 209

Graciá-Carpio, J., García-Burillo, S., Planesas, P., \& Colina, L. 2006, ApJ, 640, L135

Graciá-Carpio, J., García-Burillo, S., Planesas, P., Fuente, A., \& Usero, A. 2008, A\&A, 479, 703

Greve, T. R., Leonidaki, I., Xilouris, E. M., et al. 2014, ApJ, 794, 142

Grimes, J. P., Heckman, T., Hoopes, C., et al. 2006, ApJ, 648, 310

Hsieh, P.-Y., Ho, P. T. P., Kohno, K., Hwang, C.-Y., \& Matsushita, S. 2012, ApJ, 747, 90

Huettemeister, S., Henkel, C., Mauersberger, R., et al. 1995, A\&A, 295, 571

Ichikawa, K., Imanishi, M., Ueda, Y., et al. 2014, ApJ, 794, 139

Imanishi, M., Nakanishi, K., Tamura, Y., Oi, N., \& Kohno, K. 2007, AJ, 134, 2366

Imanishi, M., \& Nakanishi, K. 2013a, AJ, 146, 47

Imanishi, M., \& Nakanishi, K. 2013b, AJ, 146, 91

Imanishi, M., \& Nakanishi, K. 2014, AJ, 148, 9

Imanishi, M., Nakanishi, K., \& Izumi, T. 2016a, ApJ, 822, L10

Imanishi, M., Nakanishi, K., \& Izumi, T. 2016b, ApJ, 825, 44

Imanishi, M., Nakanishi, K., \& Izumi, T. 2016c, AJ, 152, 218

Imanishi, M., Nakanishi, K., \& Izumi, T. 2017, ApJ, 849, 29

Imanishi, M., Nakanishi, K., \& Izumi, T. 2018, ApJ, 856, 143

Iono, D., Ho, P. T. P., Yun, M. S., et al. 2004a, ApJ, 616, L63

Iono, D., Yun, M. S., \& Mihos, J. C. 2004b, ApJ, 616, 199

Iono, D., Wilson, C. D., Takakuwa, S., et al. 2007, ApJ, 659, 283

Iono, D., Saito, T., Yun, M. S., et al. 2013, PASJ, 65, L7

Izumi, T., Kohno, K., Martín, S., et al. 2013, PASJ, 65, 100

Izumi, T., Kohno, K., Aalto, S., et al. 2015, ApJ, 811, 39

Izumi, T., Kohno, K., Aalto, S., et al. 2016, ApJ, 818, 42

Kamenetzky, J., Rangwala, N., Glenn, J., Maloney, P. R., \& Conley, A. 2016, ApJ, 829, 93

Kazandjian, M. V., Meijerink, R., Pelupessy, I., Israel, F. P., \& Spaans, M. 2015, A\&A, 574, A127

Knudsen, K. K., Walter, F., Weiss, A., et al. 2007, ApJ, 666, 156

Kohno, K., Matsushita, S., Vila-Vilaró, B., et al. 2001, The Central Kiloparsec of Starbursts and AGN: The La Palma Connection, 249, 672

Kohno, K. 2005, The Evolution of Starbursts, 783, 203

Krips, M., Neri, R., García-Burillo, S., et al. 2008, ApJ, 677, $262-275$

Krumholz, M. R., \& McKee, C. F. 2005, ApJ, 630, 250

Krumholz, M. R., \& Thompson, T. A. 2007, ApJ, 669, 289

Lada, C. J., Forbrich, J., Lombardi, M., \& Alves, J. F. 2012, ApJ, 745,190

Lundgren, A., 2013, ALMA Cycle 2 Technical Handbook Version 1.1, ALMA

Martín, S., Kohno, K., Izumi, T., et al. 2015, A\&A, 573, A116

Mangum, J. G., \& Shirley, Y. L. 2015, PASP, 127, 266

McMullin, J. P., Waters, B., Schiebel, D., Young, W., \& Golap, K. 2007, Astronomical Data Analysis Software and Systems XVI, 376, 127

Meier, D. S., \& Turner, J. L. 2005, ApJ, 618, 259

Meijerink, R., Spaans, M., Loenen, A. F., \& van der Werf, P. P. 2011, A\&A, 525, A119

Michiyama, T., Iono, D., Nakanishi, K., et al. 2016, PASJ,

Müller, H. S. P., Thorwirth, S., Roth, D. A., \& Winnewisser, G. 2001, A\&A, 370, L49
Müller, H. S. P., Schlöder, F., Stutzki, J., \& Winnewisser, G. 2005, Journal of Molecular Structure, 742, 215

Narayanan, D., Cox, T. J., Shirley, Y., et al. 2008, ApJ, 684, 996-1008

Papadopoulos, P. P. 2007, ApJ, 656, 792

Papadopoulos, P. P., Zhang, Z.-Y., Xilouris, E. M., et al. 2014, ApJ, 788, 153

Peacock, J. A., Rowan-Robinson, M., Blain, A. W., et al. 2000, MNRAS, 318, 535

Pérez-Beaupuits, J. P., Aalto, S., \& Gerebro, H. 2007, A\&A, 476, 177

Privon, G. C., Herrero-Illana, R., Evans, A. S., et al. 2015, ApJ, 814,39

Rangwala, N., Maloney, P. R., Glenn, J., et al. 2011, ApJ, 743, 94

Rosenberg, M. J. F., van der Werf, P. P., Aalto, S., et al. 2015, ApJ, 801, 72

Saito, T., Iono, D., Yun, M. S., et al. 2015, ApJ, 803, 60

Saito, T., Iono, D., Espada, D., et al. 2017a, ApJ, 834, 6

Saito, T., Iono, D., Xu, C. K., et al. 2017b, ApJ, 835, 174

Saitoh, T. R., Daisaka, H., Kokubo, E., et al. 2009, PASJ, 61, 481

Sakamoto, K., Aalto, S., Evans, A. S., Wiedner, M. C., \& Wilner,

D. J. 2010, ApJ, 725, L228

Sakamoto, K., Aalto, S., Costagliola, F., et al. 2013, ApJ, 764, 42

Salak, D., Tomiyasu, Y., Nakai, N., et al. 2018, ApJ, 856, 97

Sandstrom, K. M., Leroy, A. K., Walter, F., et al. 2013, ApJ, 777, 5

Schirm, M. R. P., Wilson, C. D., Madden, S. C., \& Clements, D. L. 2016, ApJ, 823, 87

Shirley, Y. L. 2015, PASP, 127, 299

Scoville, N. Z., Evans, A. S., Thompson, R., et al. 2000, AJ, 119, 991

Scoville, N., Sheth, K., Aussel, H., et al. 2016, ApJ, 820, 83

Shimajiri, Y., André, P., Braine, J., et al. 2017, arXiv:1705.00213

Sliwa, K., Wilson, C. D., Petitpas, G. R., et al. 2012, ApJ, 753, 46

Sliwa, K., Wilson, C. D., Krips, M., et al. 2013, ApJ, 777, 126

Spilker, J. S., Marrone, D. P., Aguirre, J. E., et al. 2014, ApJ, 785,149

Stephens, I. W., Jackson, J. M., Whitaker, J. S., et al. 2016, ApJ, 824,29

Tateuchi, K., Konishi, M., Motohara, K., et al. 2015, ApJS, 217, 1

Teyssier, R., Chapon, D., \& Bournaud, F. 2010, ApJ, 720 , L149

Tsai, M., Hwang, C.-Y., Matsushita, S., Baker, A. J., \& Espada, D. 2012, ApJ, 746, 129

Tunnard, R., Greve, T. R., Garcia-Burillo, S., et al. 2015, ApJ, 815,114

Ueda, J., Watanabe, Y., Iono, D., et al. 2016, arXiv:1611.00002

Usero, A., Leroy, A. K., Walter, F., et al. 2015, AJ, 150, 115

Viti, S., García-Burillo, S., Fuente, A., et al. 2014, A\&A, 570, A28

Watanabe, Y., Sakai, N., Sorai, K., \& Yamamoto, S. 2014, ApJ, 788,4

Wilson, C. D., Petitpas, G. R., Iono, D., et al. 2008, ApJS, 178, $189-224$

Yun, M. S., Scoville, N. Z., \& Knop, R. A. 1994, ApJ, 430, L109

Yun, M. S., \& Carilli, C. L. 2002, ApJ, 568, 88

Zaragoza-Cardiel, J., Beckman, J., Font, J., et al. 2016, arXiv:1611.03863

Zhang, Z.-Y., Gao, Y., Henkel, C., et al. 2014, ApJ, 784, L31

Zhu, M., Seaquist, E. R., \& Kuno, N. 2003, ApJ, 588, 243

Zhu, M., Gao, Y., Seaquist, E. R., \& Dunne, L. 2007, AJ, 134, 118 
Table 1

Line and imaging properties

\begin{tabular}{|c|c|c|c|c|c|c|c|c|c|c|c|}
\hline $\begin{array}{l}\text { Line } \\
\text { (1) }\end{array}$ & $\begin{array}{l}J \\
(2)\end{array}$ & $\begin{array}{c}\nu_{\text {rest }} \\
(\mathrm{GHz}) \\
(3)\end{array}$ & $\begin{array}{c}E_{\mathrm{u}} / k \\
(\mathrm{~K}) \\
(4)\end{array}$ & $\begin{array}{l}\text { MRS } \\
\left(\begin{array}{l}\prime \prime \\
(5)\end{array}\right.\end{array}$ & $\begin{array}{c}u v \text {-weight } \\
\text { (6) }\end{array}$ & $\begin{array}{c}\text { Beam size } \\
\left(\begin{array}{c}(\prime) \\
(7)\end{array}\right.\end{array}$ & $\begin{array}{c}\Delta V \\
\left(\mathrm{~km} \mathrm{~s}_{(8)}^{-1}\right)\end{array}$ & $\begin{array}{c}\mathrm{rms} \\
\left(\mathrm{mJy} \mathrm{b} \mathrm{b}^{-1}\right) \\
(9)\end{array}$ & $\begin{array}{c}S_{\text {line }} \Delta v \\
\left(\mathrm{Jy} \mathrm{km} \mathrm{s} \mathrm{s}^{-1}\right) \\
(10)\end{array}$ & $\begin{array}{c}\text { Recovered flux } \\
(\%) \\
(11)\end{array}$ & $\begin{array}{l}\text { Ref. } \\
\text { (12) }\end{array}$ \\
\hline \multirow[t]{3}{*}{$\mathrm{HCN}$} & $1-0$ & 88.63160 & 4.25 & 22 & briggs & $1.45 \times 1.06$ & 20 & 0.4 & $3.53 \pm 0.24$ & $40 \pm 5$ & 1 \\
\hline & $3-2$ & 265.88643 & 25.52 & 9 & natural & $0.60 \times 0.47$ & 30 & 0.4 & $8.36 \pm 0.73$ & $\ldots$ & $\cdots$ \\
\hline & $4-3$ & 354.50548 & 42.53 & 7 & natural & $0.19 \times 0.15$ & 30 & 0.3 & $5.99 \pm 0.93$ & $>18$ & 2 \\
\hline \multirow{3}{*}{$\mathrm{HCO}^{+}$} & $1-0$ & 89.18852 & 4.28 & 22 & briggs & $1.45 \times 1.04$ & 20 & 0.5 & $12.18 \pm 0.69$ & $52 \pm 4$ & 1 \\
\hline & $3-2$ & 267.55763 & 25.68 & 9 & natural & $0.60 \times 0.47$ & 30 & 0.4 & $29.83 \pm 2.44$ & $\cdots$ & $\cdots$ \\
\hline & $4-3$ & 356.73422 & 42.80 & 7 & natural & $0.19 \times 0.15$ & 30 & 0.4 & $18.52 \pm 2.85$ & $46 \pm 14$ & 2 \\
\hline $\mathrm{HNC}$ & $1-0$ & 90.66357 & 4.35 & 22 & natural & $1.43 \times 1.29$ & 50 & 0.6 & $1.03 \pm 0.20$ & $27 \pm 7$ & 1 \\
\hline CS & $7-6$ & 342.88285 & 65.83 & 7 & natural & $0.18 \times 0.15$ & 50 & 0.3 & $0.41 \pm 0.10$ & $>1$ & 2 \\
\hline
\end{tabular}

Note. - Column 1: Observed molecule. Column 2: Observed rotational transition. Column 3: Rest frequency. Column 4: Upper state energy. Column 5: Maximum recoverable scale of the image cube. Column 6: Visibility (uv-plane) weighting for imaging. "briggs" means Briggs weighting with robust $=0.5$. Column 7: Beam size of the image cube. Column 8: Velocity resolution of the image cube. Column 9: Noise rms in the data which have velocity resolution of $\Delta V$. Column 10: Total integrated intensity. We consider the statistical error and the systematic error of the flux calibration in this column. Column 11: The ALMA interferometric flux divided by the single-dish flux. Column 12: Reference of the single-dish flux. (1) Privon et al. (2015); (2) Zhang et al. (2014)

Table 2

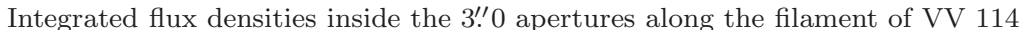

\begin{tabular}{cccccrr}
\hline \hline $\mathrm{ID}^{\mathrm{a}}$ & $\begin{array}{c}S_{\mathrm{HCN}(1-0)} d v \\
\left(\mathrm{Jm} \mathrm{s}^{-1}\right)\end{array}$ & $\begin{array}{c}S_{\mathrm{HCN}(3-2)} d v \\
\left(\mathrm{Jy} \mathrm{km} \mathrm{s}^{-1}\right)\end{array}$ & $\begin{array}{c}S_{\mathrm{HCN}(4-3)} d v \\
\left(\mathrm{Jy} \mathrm{km} \mathrm{s}^{-1}\right)\end{array}$ & $\begin{array}{c}S_{\mathrm{HCO}+(1-0)} d v \\
\left(\mathrm{Jy} \mathrm{km} \mathrm{s}^{-1}\right)\end{array}$ & $\begin{array}{c}S_{\mathrm{HCO}+(3-2)} d v \\
\left(\mathrm{Jy} \mathrm{km} \mathrm{s}^{-1}\right)\end{array}$ & $\begin{array}{c}S_{\mathrm{HCO}+(4-3)} d v \\
\left(\mathrm{Jy} \mathrm{km} \mathrm{s}^{-1}\right)\end{array}$ \\
\hline 1 & $0.38 \pm 0.06$ & $2.28 \pm 0.19$ & $2.56 \pm 0.41$ & $0.36 \pm 0.06$ & $3.33 \pm 0.27$ & $5.26 \pm 0.80$ \\
2 & $1.08 \pm 0.08$ & $4.53 \pm 0.37$ & $5.62 \pm 0.85$ & $1.46 \pm 0.10$ & $10.12 \pm 0.81$ & $16.08 \pm 2.42$ \\
3 & $1.11 \pm 0.08$ & $3.87 \pm 0.31$ & $4.57 \pm 0.70$ & $1.99 \pm 0.12$ & $10.85 \pm 0.87$ & $16.22 \pm 2.44$ \\
4 & $0.80 \pm 0.07$ & $1.88 \pm 0.16$ & $1.78 \pm 0.28$ & $1.85 \pm 0.12$ & $6.23 \pm 0.50$ & $7.62 \pm 1.15$ \\
5 & $0.58 \pm 0.07$ & $0.83 \pm 0.09$ & $<0.28$ & $1.68 \pm 0.12$ & $4.25 \pm 0.35$ & $1.96 \pm 0.32$ \\
6 & $0.58 \pm 0.07$ & $0.79 \pm 0.08$ & $0.31 \pm 0.08$ & $1.52 \pm 0.11$ & $4.32 \pm 0.35$ & $3.24 \pm 0.50$ \\
7 & $0.55 \pm 0.06$ & $0.72 \pm 0.07$ & $0.49 \pm 0.10$ & $1.19 \pm 0.09$ & $3.79 \pm 0.31$ & $4.31 \pm 0.66$ \\
8 & $0.43 \pm 0.05$ & $0.54 \pm 0.07$ & $0.27 \pm 0.08$ & $1.07 \pm 0.08$ & $2.80 \pm 0.23$ & $3.01 \pm 0.47$ \\
9 & $0.24 \pm 0.04$ & $0.34 \pm 0.05$ & $<0.27$ & $0.93 \pm 0.08$ & $2.14 \pm 0.18$ & $2.00 \pm 0.32$ \\
10 & $<0.17$ & $0.32 \pm 0.05$ & $<0.28$ & $0.71 \pm 0.07$ & $2.09 \pm 0.18$ & $1.53 \pm 0.26$ \\
11 & $<0.17$ & $<0.26$ & $<0.28$ & $0.18 \pm 0.06$ & $<0.29$ & $<0.38$ \\
\hline
\end{tabular}

${ }^{a}$ Nomenclature as in Saito et al. (2017a).

Table 3

Flux densities at "AGN" position (E0) a and "starburst" position

$(\mathrm{E} 1)^{\mathrm{a}}$

\begin{tabular}{|c|c|c|c|}
\hline & Unit & AGN (E0) & starburst (E1) \\
\hline$S_{254}$ & $\mathrm{mJy}$ & $1.50 \pm 0.12$ & . \\
\hline$S_{350}$ & mJy & $1.98 \pm 0.30$ & $1.49 \pm 0.23$ \\
\hline$S_{\mathrm{HCN}(1-0)} d v$ & $\mathrm{Jy} \mathrm{km} \mathrm{s}{ }^{-1}$ & $0.34 \pm 0.04$ & $\ldots$ \\
\hline$S_{\mathrm{HCN}(3-2)} d v$ & Jy km s ${ }^{-1}$ & $1.79 \pm 0.15$ & $\cdots$ \\
\hline$S_{\mathrm{HCN}(4-3)} d v$ & Jy km s ${ }^{-1}$ & $1.60 \pm 0.24$ & $0.62 \pm 0.10$ \\
\hline$S_{\mathrm{HCO}}+(1-0) d v$ & Jy km s${ }^{-1}$ & $0.31 \pm 0.04$ & $\ldots$ \\
\hline$S_{\mathrm{HCO}}+(3-2) d v$ & Jy km s${ }^{-1}$ & $1.53 \pm 0.13$ & $\cdots$ \\
\hline$S_{\mathrm{HCO}}+(4-3) d v$ & Jy km s${ }^{-1}$ & $1.54 \pm 0.24$ & $1.20 \pm 0.18$ \\
\hline$S_{\mathrm{HNC}(1-0)} d v$ & Jy km s${ }^{-1}$ & $0.29 \pm 0.07$ & $\cdots$ \\
\hline$S_{\mathrm{CS}(7-6)} d v$ & Jy km s${ }^{-1}$ & $0.33 \pm 0.07$ & $<0.12$ \\
\hline$S_{\mathrm{HCN}\left(\nu_{2}=1^{\mathrm{f}}, 3-2\right)} d v$ & Jy km s${ }^{-1}$ & $<0.26$ & $\cdots$ \\
\hline$S_{\mathrm{HCN}\left(\nu_{2}=1^{\mathrm{f}}, 4-3\right)} d v$ & Jy km s${ }^{-1}$ & $<0.26$ & $<0.12$ \\
\hline
\end{tabular}

a positions defined by Iono et al. (2013) 
Table 4

$\Sigma_{\text {dense }}, f_{\text {dense }}, \mathrm{SFE}_{\mathrm{H}_{2}}$, and $\mathrm{SFE}_{\text {dense }}$

\begin{tabular}{|c|c|c|c|c|}
\hline (1) & $\begin{array}{c}\Sigma_{\text {dense }} \\
\left(M_{\odot} \mathrm{pc}^{-2}\right) \\
(2)\end{array}$ & $\begin{array}{c}f_{\text {dense }} \\
(3) \\
\end{array}$ & $\begin{array}{c}\mathrm{SFE}_{\mathrm{H}_{2}} \\
\left(\mathrm{Myr}^{-1}\right) \\
(4)\end{array}$ & $\begin{array}{c}\mathrm{SFE}_{\text {dense }} \\
\left(\mathrm{Myr}^{-1}\right) \\
(5)\end{array}$ \\
\hline 1 & $90 \pm 13$ & $13 \pm 0.02$ & $0.038 \pm 0.004$ & $0.28 \pm 0.05$ \\
\hline 2 & $260 \pm$ & \pm 0 & $0.040 \pm$ & $0.22=$ \\
\hline J & $268 \pm 1$ & & $7 \pm$ & 0.19 \\
\hline 4 & $192 \pm 17$ & \pm & $0.023 \pm$ & $0.13=$ \\
\hline 5 & $139 \pm$ & \pm & $7 \pm$ & $0.10=$ \\
\hline 6 & $140 \pm 18$ & $22 \pm 0$ & $0.017 \pm$ & $0.08 \pm 0.01$ \\
\hline 7 & $132 \pm 14$ & 1 & $0.016 \pm$ & $0.08 \pm$ \\
\hline 8 & $104 \pm 1$ & 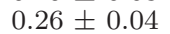 & 4 & $0.06 \pm$ \\
\hline 9 & $59 \pm 11$ & $0.26 \pm 0.06$ & $0.020 \pm 0.006$ & $0.08 \pm 0.03$ \\
\hline 10 & $<40$ & $<0.17$ & $0.046 \pm$ & $<0.28$ \\
\hline 11 & $<41$ & $<0.20$ & $0.047 \pm 0.011$ & $<0.23$ \\
\hline
\end{tabular}

Column 1: Nomenclature as in Saito et al. (2017a). Column 2: Dense gas mass surface density derived from the HCN (1-0) integrated intensity. Column 3: Dense gas fraction $\left(=\Sigma_{\text {dense }} / \Sigma_{\mathrm{H}_{2}}\right)$. We used $N_{\mathrm{H}_{2}}$ as in Saito et al. (2017a), and converted to $\Sigma_{\mathrm{H}_{2}}$. Column 4: Star formation efficiency $\left(=\Sigma_{\mathrm{SFR}} / \Sigma_{\mathrm{H}_{2}}\right)$. We used $\Sigma_{\mathrm{SFR}}$ as in Saito et al. (2017a). Column 5: Dense gas star formation efficiency $\left(=\Sigma_{\text {SFR }} / \Sigma_{\text {dense }}\right)$

Table 5

Results of rotation diagram

\begin{tabular}{lccccccc}
\hline \hline $\mathrm{ID}^{\mathrm{a}}$ & $\begin{array}{c}T_{\mathrm{HCN}} \\
(\mathrm{K})\end{array}$ & $\begin{array}{c}N_{\mathrm{HCN}} \\
\left(\mathrm{cm}^{-2}\right)\end{array}$ & $\begin{array}{c}X_{\mathrm{HCN}} \\
\left(10^{-9}\right)\end{array}$ & $\begin{array}{c}T_{\mathrm{HCO}+} \\
(\mathrm{K})\end{array}$ & $\begin{array}{c}N_{\mathrm{HCO}} \\
\left(\mathrm{cm}^{-2}\right)\end{array}$ & $\begin{array}{c}X_{\mathrm{HCO}}+ \\
\left(10^{-9}\right)\end{array}$ & $X_{\mathrm{HCN}} / X_{\mathrm{HCO}}{ }^{\mathrm{b}}$ \\
\hline 1 & $10.2 \pm 2.5$ & $(1.0 \pm 0.6) \times 10^{14}$ & $2.0 \pm 1.3$ & $12.3 \pm 2.8$ & $(9.4 \pm 5.0) \times 10^{13}$ & $2.0 \pm 1.1$ & $1.0 \pm 0.9$ \\
2 & $8.6 \pm 2.0$ & $(3.1 \pm 1.9) \times 10^{14}$ & $3.2 \pm 2.0$ & $10.0 \pm 1.7$ & $(4.5 \pm 1.7) \times 10^{14}$ & $4.6 \pm 1.8$ & $0.7 \pm 0.5$ \\
3 & $8.1 \pm 1.7$ & $(3.3 \pm 1.9) \times 10^{14}$ & $3.3 \pm 1.9$ & $9.2 \pm 1.5$ & $(6.1 \pm 2.2) \times 10^{14}$ & $6.3 \pm 2.3$ & $0.5 \pm 0.4$ \\
4 & $7.2 \pm 1.6$ & $(2.2 \pm 1.6) \times 10^{14}$ & $3.0 \pm 2.2$ & $7.7 \pm 1.3$ & $(5.6 \pm 2.4) \times 10^{14}$ & $7.6 \pm 3.3$ & $0.4 \pm 0.3$ \\
5 & $5.2 \pm 1.5$ & $(2.1 \pm 1.3) \times 10^{14}$ & $3.7 \pm 2.3$ & $6.4 \pm 0.5$ & $(5.4 \pm 1.3) \times 10^{14}$ & $9.5 \pm 2.5$ & $0.4 \pm 0.3$ \\
6 & $5.7 \pm 0.7$ & $(1.8 \pm 0.9) \times 10^{14}$ & $4.0 \pm 2.0$ & $7.0 \pm 0.8$ & $(4.7 \pm 1.7) \times 10^{14}$ & $10.5 \pm 4.0$ & $0.4 \pm 0.2$ \\
7 & $6.0 \pm 1.2$ & $(1.6 \pm 1.2) \times 10^{14}$ & $3.3 \pm 2.5$ & $7.6 \pm 1.3$ & $(3.5 \pm 1.7) \times 10^{14}$ & $7.5 \pm 3.7$ & $0.4 \pm 0.4$ \\
8 & $5.7 \pm 0.8$ & $(1.3 \pm 0.7) \times 10^{14}$ & $4.9 \pm 2.6$ & $7.1 \pm 1.2$ & $(3.2 \pm 1.6) \times 10^{14}$ & $11.5 \pm 5.9$ & $0.4 \pm 0.3$ \\
9 & $5.2 \pm 1.5$ & $(8.6 \pm 5.4) \times 10^{13}$ & $5.2 \pm 3.4$ & $6.8 \pm 1.1$ & $(2.7 \pm 1.4) \times 10^{14}$ & $16.5 \pm 9.0$ & $0.3 \pm 0.3$ \\
10 & $6.2^{\mathrm{c}}$ & $(4.3 \pm 0.7) \times 10^{13}$ & $2.6 \pm 5.6$ & $7.1 \pm 0.8$ & $(2.1 \pm 0.8) \times 10^{14}$ & $1.3 \pm 5.1$ & $0.2 \pm 0.1$ \\
11 & $6.2^{\mathrm{c}}$ & $<3.5 \times 10^{13}$ & $<2.6$ & $7.4^{\mathrm{c}}$ & $\cdots \cdot \mathrm{d}$ & $\cdots$ & $\mathrm{d}$ \\
$\mathrm{E} 0$ & $9.8 \pm 1.6$ & $(8.0 \pm 3.9) \times 10^{14}$ & $\cdots$ & $10.9 \pm 2.4$ & $(5.0 \pm 3.0) \times 10^{14}$ & $\cdots$ & $1.5 \pm 1.2$ \\
\hline
\end{tabular}

a Aperture ID defined by Saito et al. (2017a). E0 is the AGN position at the eastern nucleus defined by Iono et al. (2013).

b This is derived by $N_{\mathrm{HCN}} / N_{\mathrm{HCO}}+$ in practice.

${ }^{c}$ In the case of non-detection or single transition detection, we apply the average $T_{\text {rot }}$ between Region 3 and 9 to derive $N_{\text {tot }}$. See text.

${ }^{d}$ We did not estimate $N_{\mathrm{HCO}}+$ and $X_{\mathrm{HCO}}+$ at Region 11 because we only marginally detected the $\mathrm{HCO}^{+}(1-0)$ line. 